hep-ph/9501280

CHIBA-EP-85

DPNU-94-51

\title{
Spontaneous Chiral-Symmetry Breaking in Three-Dimensional QED with a Chern-Simons Term
}

\author{
K.-I. Kondd* \\ Department of Physics, Chiba University, Chiba 263, Japan \\ P. Marisi \\ Department of Physics, Nagoya University, Nagoya 464-01, Japan
}

(December 16, 1994)

\begin{abstract}
In three-dimensional QED with a Chern-Simons term we study the phase structure associated with chiral-symmetry breaking in the framework of the Schwinger-Dyson equation. We give detailed analyses on the analytical and numerical solutions for the Schwinger-Dyson equation of the fermion propagator, where the nonlocal gauge-fixing procedure is adopted to avoid wavefunction renormalization for the fermion. In the absence of the Chern-Simons term, there exists a finite critical number of four-component fermion flavors, at which a continuous (infinite-order) chiral phase transition takes place and below which the chiral symmetry is spontaneously broken. In the presence of the Chern-Simons term, we find that the spontaneous chiral-symmetrybreaking transition continues to exist, but the type of phase transition turns into a discontinuous first-order transition. A simple stability argument is given based on the effective potential, whose stationary point gives the solution of the Schwinger-Dyson equation.
\end{abstract}

11.30.Qc, 11.30.Rd, 11.10.Kk, 11.15.Tk

Typeset using REVTEX 


\section{INTRODUCTION}

This paper is the detailed exposition of our previous letter [1] on chiral-symmetry breaking in (2+1)-dimensional QED with $N$ flavors of four-component Dirac fermions (hereafter called QED3). Such a model of QED3 is chirally symmetric in the absence of a bare fermion mass term, $m_{0} \bar{\psi} \psi$, in sharp contrast to the $(2+1)$-dimensional gauge theory with two-component fermions, where we cannot define chiral symmetry [2] Similarly to the four-dimensional case [5], the chiral symmetry of QED3 may be broken spontaneously due to the dynamical generation of a fermion mass. However the pattern of dynamical symmetry breaking is shown to be qualitatively different from the four-dimensional counterparts.

Recently, QED3 has found a vast region of application in condensed matter physics as an effective theory in the long wavelength (or low-energy) limit of a more realistic microscopic model [7 9]. Especially, since the discovery of high- $T_{c}$ superconductivity and fractional quantum Hall effect, a peculiarity of three-dimensional gauge theory, i.e., the existence of a Chern-Simons (CS) term [6]

$$
\frac{\theta}{2} \epsilon^{\mu \nu \rho} A_{\mu} \partial_{\nu} A_{\rho}
$$

has been a basic ingredient in these applications. The CS term gives the gauge field a mass without destroying the gauge invariance. And, even if there is no bare CS term in the original Lagrangian, a CS term can be generated by radiative corrections in three dimensions. In the presence of a CS term, parity is broken explicitly, but the Lagrangian still keeps the chiral symmetry when $m_{0} \bar{\psi} \psi=0$. The parity violation is a very important factor for the anyonic model to be a candidate theory of high- $T_{c}$ superconductivity [7,8]. A field-theoretic realization of such an anyonic model consists of fermions interacting with an abelian statistical gauge field whose dynamics is governed by a CS term. In contrast to the chiral symmetry, it is known that the dynamical breakdown of parity does not occur in QED3 [10 [16].

In this paper we pay attention to the breaking of chiral symmetry in the presence of a CS term within the framework of the Schwinger-Dyson (SD) equation [17]. In momentum space, the $\mathrm{SD}$ equation for the full fermion propagator $S(p)$ is written as

$$
S^{-1}(p)=p_{\mu} \gamma_{\mu}-m_{0}-e^{2} \int \frac{d^{3} q}{(2 \pi)^{3}} \gamma_{\mu} S(q) \Gamma_{\nu}(q, p) D_{\mu \nu}(k),
$$

which should be solved self-consistently and simultaneously with other SD equations for the full photon propagator $D_{\mu \nu}(k)$ and the full vertex function $\Gamma_{\mu}(q, p)$. In order to solve these sets of SD equations actually, however, we adopt some kind of approximations as exemplified below.

There are several quantities we must specify to write down the closed SD equation for the fermion propagator $S(p)$, namely, the full photon propagator $D_{\mu \nu}(k)$ and the vertex function $\Gamma_{\mu}(p, q)$, and we also have to choose a suitable gauge. In general, both the photon propagator and the vertex satisfy their own SD equation, and both depend on the gauge-

fixing parameter $a$, if the covariant gauge-fixing Lagrangian $\frac{1}{2 a}\left(\partial_{\mu} A^{\mu}\right)^{2}$ is taken. There are also additional constraints on these $n$-point functions, coming from relations such as the Ward-Takahashi (WT) identity 


$$
\left(p_{\mu}-q_{\mu}\right) \Gamma^{\mu}(p, q)=S^{-1}(p)-S^{-1}(q) .
$$

Now one of the problems in QED3 is to find a consistent truncation scheme for the SD equation: as a consequence of the WT identity the wave-function renormalization should be equal to the vertex renormalization, which puts a constraint on the bare vertex approximation. By using

$$
S(p)=\left[\gamma_{\mu} p_{\mu} A(p)-B(p)\right]^{-1},
$$

the SD equation for $S(p)$ is decomposed into two integral equations for $A(p)$, the wavefunction renormalization, and $B(p)$. Although with a bare vertex it is not possible to satisfy the WT identity exactly, we should make sure to satisfy the constraint $A(p) \simeq 1$ when using the bare vertex approximation.

First of all, we restrict our attention to QED without CS term. In the quenched case, in which the vacuum polarization to the photon propagator is completely neglected, the simplest treatment is to choose the Landau gauge $a=0$ and to take the bare vertex $\Gamma_{\mu}(p, q) \equiv \gamma_{\mu}$. This quenched approximation is equivalent to taking the $N \rightarrow 0$ limit in the $\mathrm{SD}$ equation for the fermion propagator, since this limit eliminates the radiative correction to the photon propagator coming from the internal fermion loops (with $N$ species). Then the above SD equation leads to no wave-function renormalization: $A(p) \equiv 1$, irrespective of $B(p)$, see e.g. [18]. In this case, it has been shown [19] that quenched QED3 resides in only one phase where chiral symmetry is spontaneously broken, in agreement with the Monte Carlo simulation of non-compact lattice QED3 [20].

In the quenched case, the bare vertex approximation is justified in the Landau gauge $a=0$, because this choice is approximately consistent with the WT identity and satisfies the requirement that the wave-function renormalization and the vertex renormalization are equal. In gauges other than the Landau gauge however, we can not take the bare vertex, since $A(p)$ deviates from one. In order to study the SD equation in a general gauge, we must modify the vertex in such a way that the result is gauge-covariant, i.e., independent of the gauge-fixing parameter $a$. This problem persists in the three-dimensional case [21,22] as well as in four dimensions [23 25]. Such a modified vertex has to satisfy the WT identity exactly.

Now we can raise the question whether the chiral symmetry restores at a certain nonzero value of $N$. In the presence of the one-loop vacuum polarization $(N \neq 0)$ the simple procedure mentioned above can not be applied, since there is no simple gauge choice $a$ such that $A(p) \equiv 1$. Nevertheless, under the bare vertex approximation in the Landau gauge, Appelquist, Nash and Wijewardhana (ANW) [27] have shown that there is a finite critical number of flavors $N_{c}$, above which the chiral symmetry restores. Based on a leading-order $1 / N$ expansion [26] ( $e^{2}=8 \alpha / N$ with $\alpha$ being kept fixed) for the vertex and the photon propagator in the SD equation, they gave the critical value $N_{c}=32 / \pi^{2} \cong 3.2$. Furthermore, Nash [28] claimed that the leading-order gauge-invariant critical number of flavors is given by $N_{c}=128 / 3 \pi^{2}$ and that, when $1 / N^{2}$ corrections are included, $N_{c}$ coincides approximately with this result.

However, such a simple treatment of the vertex function and the wave-function renormalization function $A$ was criticised by Pennington and Webb [29] and Atkinson, Johnson and Pennington [30]. They claimed that, if the vertex is correctly improved, using the 
WT identity, and the coupled equations for $A(p)$ and $B(p)$ are solved together, the finite critical number of flavors does not exist. This implies that the chiral symmetry is spontaneously broken in QED3 for all values of $N$, i.e., $N_{c}=\infty$. On the other hand, the Monte Carlo simulation [20] of lattice non-compact QED3 seems to support a finite critical flavors: 1 $N_{c} \sim 3.5 \pm 0.5$, see also [31]. However, it is numerically very difficult to confirm the exponential decrease of the dynamical mass for increasing $N$ found in 29, 30.

The origin of this controversy stems from the fact that in the unquenched case $A(p) \equiv 1$ can not be deduced as a simple consequence of the SD equation by choosing the Landau gauge, in sharp contrast with the quenched case. Nakatani [32] has proposed to use a nonlocal and momentum-dependent gauge function, instead of the usual gauge-fixing term, in order to keep $A(p) \equiv 1$ and thus to overcome the inconsistency one has using the bare vertex approximation in the Landau gauge. Actually the nonlocal gauge found in [33, 34, 16] can play exactly the same role as the Landau gauge in the quenched case, in the sense that $A(p) \equiv 1$ follows by choosing the appropriate nonlocal gauge in the bare vertex approximation and that the nonlocal gauge $a(k)$ as a function of the photon momentum $k$ reduces to the Landau gauge in the quenched limit $N \rightarrow 0$. Taking into account only the leading and next-to-leading order terms in the infrared region, which is the essential region in QED3 [2.35], yields a finite critical number of flavors [16]

$$
N_{c}=128 / 3 \pi^{2} \cong 4.3,
$$

which is the same as the leading-order result obtained by Nakatani, and coincides with the result obtained by Nash in a different way [28]. In this paper we use the nonlocal gauge as derived in 16.

In the present paper the existence of a finite critical number of flavors is confirmed by solving numerically the nonlinear SD equation, using a bare vertex and the nonlocal gauge as derived in [16] in order to keep $A(p) \equiv 1$, without further approximation. The parityconserving and chiral-symmetry-breaking fermion mass is dynamically generated in QED3 and there occurs a chiral-symmetry-restoring phase transition at $N_{c}=128 /\left(3 \pi^{2}\right)$. The order of the chiral phase transition is infinite in the sense that the dynamical fermion mass $m_{d}$ and the chiral order parameter $\langle\bar{\psi} \psi\rangle$ exhibit an essential singularity at the critical point $N=N_{c}$,

$$
\frac{\langle\bar{\psi} \psi\rangle}{\alpha^{2}} \sim\left(\frac{m_{d}}{\alpha}\right)^{3 / 2} \sim \exp \left[-\frac{3 \pi}{\sqrt{N_{c} / N-1}}\right]
$$

In the presence of CS term, as already reported in [1], the chiral phase transition turns into a first-order transition, in sharp contrast to the $\theta=0$ case. This result is obtained by both a numerical study of the full SD equation and an analytical study of approximated equations using the same scheme as in the absence of CS term. We have also determined the critical line $N=N_{c}(\theta)$ for this transition in the $(N, \theta)$ plane, the two-dimensional

\footnotetext{
${ }^{1}$ The $N \rightarrow \infty$ limit (with $\alpha$ being kept finite) corresponds to the weak-coupling limit $\beta:=$ $1 /\left(\epsilon e^{2}(\epsilon)\right) \rightarrow \infty$ in the lattice gauge theory defined on a lattice with lattice spacing $\epsilon$.
} 
phase diagram. In this paper we give the detailed exposition of this result [1] based on the framework of [16]. The first-order transition implies that the chiral order parameter as well as the dynamical fermion mass show a discontinuous change at the critical point on the whole critical line extending from $(N, \theta)=\left(N_{c}, 0\right)$ in the phase diagram $(N, \theta)$. Moreover, the critical number of flavors $N_{c}(\theta)$ decreases as the CS coefficient $\theta$ increases.

This paper is organized as follows. In section II we give a description of QED3 with a CS term. We start from the formulation of QED3 using four-component fermions in the reducible representation of the Clifford algebra for the $\gamma$-matrices in $(2+1)$-dimensions. We define the chiral and the parity transformations for this theory, and our decomposition of the fermion propagator into scalar functions. We also discuss the structure of the gauge boson propagator with the nonlocal gauge and give an explicit expression for the vacuum polarization in the leading order of $1 / N$ expansion. After these preliminaries, we first discuss QED without the CS term and subsequently study the effect of the explicit CS term. In section IIII we write down the SD equation for the fermion propagator explicitly. The SD equation is solved both analytically and numerically. Next, in section $\mathbb{\square}$, we study the effect of the explicit CS term by solving the nonlinear SD equation numerically. In section $\square$ detailed comparison of numerical and analytical result is given and the numerical results in section $\mathbb{I V}$ are confirmed by the analytical treatment. In order to study the stability of the chiral-symmetry-breaking solution, we evaluate the effective potential of Cornwall-JackiwTomboulis [36] in section V1. The final section is devoted to conclusion and discussion. In Appendix A, we give integration formulae which are necessary to perform the angular integration to obtain the integration kernel in the SD equation. In Appendix B, we supplement details on the calculation of the ultraviolet boundary condition. In Appendix C, it is shown that at the stationary point the effective potential obtained in section $\mathrm{V} \mathbb{1}$ actually gives the $\mathrm{SD}$ equation for the fermion mass function in the nonlocal gauge.

\section{QED3 WITH CHERN-SIMONS TERM}

In Euclidean space the Lagrangian is

$$
\mathcal{L}=\bar{\psi}\left(i \not \partial+e \not A+m_{e}+\tau m_{o}\right) \psi+\frac{1}{4} F_{\mu \nu}^{2}-\frac{1}{2} i \theta \epsilon_{\mu \nu \rho} A_{\mu} \partial_{\nu} A_{\rho}+\mathcal{L}_{\text {gauge fixing }} .
$$

We use four-component spinors for the fermions, and accordingly a four-dimensional representation for the $\gamma$-matrices of the Clifford algebra $\left\{\gamma_{\mu}, \gamma_{\nu}\right\}=-2 \delta_{\mu \nu}$ :

$$
\gamma_{0} \equiv\left(\begin{array}{cc}
-i \sigma^{3} & 0 \\
0 & i \sigma^{3}
\end{array}\right), \quad \gamma_{1} \equiv\left(\begin{array}{cc}
i \sigma^{1} & 0 \\
0 & -i \sigma^{1}
\end{array}\right), \quad \gamma_{2} \equiv\left(\begin{array}{cc}
i \sigma^{2} & 0 \\
0 & -i \sigma^{2}
\end{array}\right) \text {, }
$$

with $\sigma_{a}(a=1,2,3)$ being the Pauli matrices; furthermore we have the matrix $\tau$, defined by

$$
\tau=\left(\begin{array}{cc}
1 & 0 \\
0 & -1
\end{array}\right) .
$$

With such a representation we can define chirality just as in four-dimensional QED, but now there are two independent chiral transformations possible, which are defined by the $4 \times 4$ matrices $\gamma_{3}$ and $\gamma_{5}$ : 


$$
\gamma_{3} \equiv\left(\begin{array}{ll}
0 & 1 \\
1 & 0
\end{array}\right), \quad \gamma_{5} \equiv \gamma_{0} \gamma_{1} \gamma_{2} \gamma_{3}=\left(\begin{array}{cc}
0 & -1 \\
1 & 0
\end{array}\right)
$$

Without an explicit mass $m_{e}$ for the fermions, the Lagrangian is chirally symmetric, but the mass term $m_{e} \bar{\psi} \psi$ breaks chiral symmetry. Note that the other mass term, $m_{o} \bar{\psi} \tau \psi$, is chirally invariant.

In this representation, the parity transformation is defined by $\psi\left(x_{0}, x_{1}, x_{2}\right) \rightarrow$ $P \psi\left(x_{0},-x_{1}, x_{2}\right), A_{\mu}\left(x_{0}, x_{1}, x_{2}\right) \rightarrow(-1)^{\delta_{\mu 1}} A_{\mu}\left(x_{0},-x_{1}, x_{2}\right)$, with $P=-i \gamma_{5} \gamma_{1}$. Then the mass term $m_{o} \bar{\psi} \tau \psi$ is odd under a parity transformation. Also the CS term is odd under this parity transformation, so in the presence of a (bare) CS term parity is always broken, even if $m_{o}=0$. The other terms in the Lagrangian, including the chiral-symmetry-breaking mass term, are invariant under a parity transformation.

\section{A. The Fermion Propagator}

The inverse full fermion propagator can be written as

$$
S^{-1}(p)=A_{e}(p) \not p+A_{o}(p) \tau \not p-B_{e}(p)-B_{o}(p) \tau .
$$

The functions $A(p)$ and $B(p)$ are scalar functions of the absolute values of the momenta, and their bare values are $A_{e}=1, A_{0}=0, B_{e}=m_{e}$, and $B_{o}=m_{o}$. In order to study the fermion propagator nonperturbatively it is useful to define the matrix (projection operator)

$$
\chi_{ \pm}=\frac{1}{2}(1 \pm \tau)
$$

which allows us to rewrite the full propagator as

$$
\begin{aligned}
S(p) & =S_{+}(p) \chi_{+}+S_{-}(p) \chi_{-} \\
& =-\frac{A_{+}(p) \not p+B_{+}(p)}{A_{+}^{2}(p) p^{2}+B_{+}^{2}(p)} \chi_{+}-\frac{A_{-}(p) \not p+B_{-}(p)}{A_{-}^{2}(p) p^{2}+B_{-}^{2}(p)} \chi_{-}
\end{aligned}
$$

where we have defined

$$
A_{ \pm}=A_{e} \pm A_{o}
$$

and

$$
B_{ \pm}=B_{e} \pm B_{o}
$$

In this paper we study the dynamical breaking of chiral symmetry, using the SD equation for the fermion propagator. Perturbatively this will not happen, but just as in pure QED (without CS term), chiral symmetry can be broken dynamically due to nonperturbative effects, starting with a chirally symmetric Lagrangian. So in the remainder we have put both explicit masses $m_{e}$ and $m_{o}$ equal to zero 2 , and study the behavior of $B_{ \pm}$nonperturbatively.

\footnotetext{
${ }^{2}$ We will reconsider this procedure in section VI.
} 
Note that in terms of $B_{+}$and $B_{-}$, chiral symmetry means $B_{+}(p)=-B_{-}(p)$, which gives $B_{e}(p)=0$.

The order parameter connected with the chiral phase transition is the chiral condensate. In the presence of a CS term there are two types of condensates: a parity even condensate $\langle\bar{\psi} \psi\rangle$ and a parity odd condensate $\langle\bar{\psi} \tau \psi\rangle$. Using the decomposition of the propagator in terms of $\chi_{ \pm}$, we can write these condensates as

$$
\begin{aligned}
\langle\bar{\psi} \psi\rangle & =\langle\bar{\psi} \psi\rangle_{+}+\langle\bar{\psi} \psi\rangle_{-}, \\
\langle\bar{\psi} \tau \psi\rangle & =\langle\bar{\psi} \psi\rangle_{+}-\langle\bar{\psi} \psi\rangle_{-},
\end{aligned}
$$

where

$$
\langle\bar{\psi} \psi\rangle_{ \pm}=\frac{1}{\pi^{2}} \int_{0}^{\infty} \mathrm{d} k \frac{k^{2} B_{ \pm}(k)}{A_{ \pm}^{2}(k) k^{2}+B_{ \pm}^{2}(k)} .
$$

The general SD equation is given by

$$
\begin{aligned}
& B_{ \pm}\left(p^{2}\right)=e^{2} \int \frac{\mathrm{d}^{3} k}{(2 \pi)^{3}} \frac{1}{2} \operatorname{Tr}\left[\gamma_{\mu} S_{ \pm}(k) \Gamma_{\nu}(p, k) D_{\mu \nu}(p-k) \chi_{ \pm}\right] \\
& A_{ \pm}\left(p^{2}\right)=1+\frac{e^{2}}{p^{2}} \int \frac{\mathrm{d}^{3} k}{(2 \pi)^{3}} \frac{1}{2} \operatorname{Tr}\left[\not p \gamma_{\mu} S_{ \pm}(k) \Gamma_{\nu}(p, k) D_{\mu \nu}(p-k) \chi_{ \pm}\right] .
\end{aligned}
$$

In QED3, the usual truncation scheme for the fermion SD equation is based on the $1 / N$ expansion [26]. The coupling constant $e^{2}$ has the dimension of mass, and we use the large $N$ limit in such a way that $e^{2} \downarrow 0$ and the product $N e^{2}$ remains fixed:

$$
e^{2}=8 \alpha / N
$$

with $\alpha$ fixed. In this approximation scheme, the full vertex is replaced by the bare vertex, because that is the leading-order contribution in $1 / N$. In order to be consistent with the WT identity, or at least with the requirement that the vertex renormalization and the fermion wave-function renormalization are equal, we use a suitable nonlocal gauge function.

\section{B. The Gauge Boson Propagator}

We use a Lagrangian with a so called nonlocal gauge-fixing term for the gauge field, namely

$$
\mathcal{L}(A ; f(x-y))=\frac{1}{4} F_{\mu \nu}^{2}-\frac{1}{2} i \theta \epsilon_{\mu \nu \rho} A_{\mu} \partial_{\nu} A_{\rho}+\frac{1}{2} \int \mathrm{d}^{3} y f(x-y) \partial_{\mu} A_{\mu}(x) \partial_{\nu} A_{\nu}(y),
$$

which has certain advantages above the normal gauge-fixing term: it allows for a momentumdependent gauge parameter in the gauge boson propagator.

It is easy to show that this Lagrangian leads to the following inverse bare photon propagator

$$
D_{\mu \nu}^{0-1}(q)=q^{2}\left(\delta_{\mu \nu}-\frac{q_{\mu} q_{\nu}}{q^{2}}\right)+\theta \epsilon_{\mu \nu \rho} q_{\rho}+a^{-1}(q) q_{\mu} q_{\nu}
$$


where we have defined the momentum-dependent gauge parameter in the following way

$$
a^{-1}(q)=\int \mathrm{d}^{3} x f(x) e^{-i q x} .
$$

So if we can simplify the actual calculations by choosing a specific momentum-dependent gauge $a(q)$, we can justify this afterwards by specifying the gauge-fixing term as

$$
\mathcal{L}=\frac{1}{2} \int \mathrm{d}^{3} y f(x-y) \partial_{\mu} A_{\mu}(x) \partial_{\nu} A_{\nu}(y),
$$

with

$$
f(x)=\int \frac{\mathrm{d}^{3} q}{(2 \pi)^{3}} a^{-1}(q) e^{i q x} .
$$

The conventional (local) gauge can be recovered by the choice $f(x)=\delta(x) / a$, as can easily be seen from the above formulae.

We can show that identities relating the different Green's functions, like the WT identity, hold with this nonlocal gauge in exactly the same way as with a constant gauge parameter. For the (full) photon propagator this implies in momentum space

$$
q^{2} q^{\mu} D_{\mu \nu}(q)=-a(q) q_{\nu} .
$$

This means that also with this nonlocal gauge the gauge-dependent part of the photon propagator is not affected by the interactions, and that the longitudinal part of the vacuum polarization is zero. Therefore we can write the full photon propagator as

$$
\begin{aligned}
D_{\mu \nu}(q) & =D^{T}\left(q^{2}\right)\left(\delta_{\mu \nu}-\frac{q_{\mu} q_{\nu}}{q^{2}}\right)+D^{O}\left(q^{2}\right) \epsilon_{\mu \nu \rho} \frac{q_{\rho}}{|q|}+a(q) \frac{q_{\mu} q_{\nu}}{q^{4}}, \\
D^{T}\left(q^{2}\right) & =\frac{q^{2}-\Pi^{T}(q)}{\left(q^{2}-\Pi^{T}(q)\right)^{2}+\left(\Pi^{O}(q)-\theta|q|\right)^{2}}, \\
D^{O}\left(q^{2}\right) & =\frac{\Pi^{O}(q)-\theta|q|}{\left(q^{2}-\Pi^{T}(q)\right)^{2}+\left(\Pi^{O}(q)-\theta|q|\right)^{2}},
\end{aligned}
$$

where $\Pi^{T}$ and $\Pi^{O}$ correspond to the decomposition of the vacuum polarization tensor [6]:

$$
\Pi_{\mu \nu}(q)=\left(\delta_{\mu \nu}-\frac{q_{\mu} q_{\nu}}{q^{2}}\right) \Pi^{T}(q)+\epsilon_{\mu \nu \rho} \frac{q_{\rho}}{|q|} \Pi^{O}(q) .
$$

In the $1 / N$ expansion the one-loop vacuum polarization has to be taken into account, because this vacuum polarization is of order one: there are $N$ fermion loops contributing to the vacuum polarization and each loop is of the order $e^{2} \sim 1 / N$. Starting with massless fermions, e.g. both the parity odd and parity even mass equal to zero, there is no parity odd part of the vacuum polarization. The transverse part of the vacuum polarization is

$$
\Pi_{T}(q)=-\alpha|q| .
$$

Therefore the inclusion of this vacuum polarization leads to the following gauge boson propagator 


$$
\begin{aligned}
& D^{T}\left(q^{2}\right)=\frac{q^{2}+\alpha|q|}{q^{2}\left((|q|+\alpha)^{2}+\theta^{2}\right)}, \\
& D^{O}\left(q^{2}\right)=\frac{-\theta|q|}{q^{2}\left((|q|+\alpha)^{2}+\theta^{2}\right)} .
\end{aligned}
$$

This is the photon propagator we will use in this paper, with a suitable choice for $a(q)$ in order to keep the wave-function renormalization equal to one.

\section{DYNAMICAL SYMMETRY BREAKING IN PURE QED}

Without the CS term in the Lagrangian, we have no explicit parity breaking terms, and the full fermion propagator will also be parity even: there will be no spontaneous breaking of parity [10 16]. That means that we only have to deal with one set of two coupled integral equations for $A(p)$ and $B(p)$

$$
\begin{aligned}
A\left(p^{2}\right)= & 1+\frac{8 \alpha}{N p^{2}} \int \frac{\mathrm{d}^{3} k}{(2 \pi)^{3}} \frac{A(k)}{k^{2} A^{2}(k)+B^{2}(k)} \times \\
& \left(\left(D^{T}(q)-\frac{a(q)}{q^{2}}\right) \frac{2(p \cdot q)(k \cdot q)}{q^{2}}+\frac{a(q) p \cdot k}{q^{2}}\right), \\
B\left(p^{2}\right)= & \frac{8 \alpha}{N} \int \frac{\mathrm{d}^{3} k}{(2 \pi)^{3}} \frac{B(k)}{k^{2} A^{2}(k)+B^{2}(k)}\left(2 D^{T}(q)+\frac{a(q)}{q^{2}}\right),
\end{aligned}
$$

with $q=k-p$.

The condition that the wave-function renormalization is equal to one leads to [16]

$$
a(q)=2 q^{2} D^{T}(q)+\frac{4 \alpha}{|q|}-2-\frac{4 \alpha^{2}}{q^{2}} \ln \left(\frac{\alpha+|q|}{\alpha}\right) .
$$

The SD equation for the fermions thus reduces to only one nonlinear integral equation for the dynamical mass function, which we call $m(p)$. After the angular integration, which can be done analytically (see appendix A), the radial integration kernel becomes

$$
\begin{aligned}
\mathrm{K}(p, k)= & \frac{2 \alpha}{\max (k, p)\left|k^{2}-p^{2}\right|}+\frac{1}{k p} \ln \left(\frac{\alpha+|k+p|}{\alpha+|k-p|}\right) \\
& -\frac{\alpha^{2}}{k p(k-p)^{2}} \ln \left(1+\frac{|k-p|}{\alpha}\right)+\frac{\alpha^{2}}{k p(k+p)^{2}} \ln \left(1+\frac{|k+p|}{\alpha}\right),
\end{aligned}
$$

and the equation for the mass function is

$$
m(p)=\frac{4 \alpha}{\pi^{2} N} \int_{0}^{\infty} \mathrm{d} k \frac{k^{2} m(k)}{k^{2}+m^{2}(k)} \mathrm{K}(p, k) .
$$

This nonlinear integral equation can be solved numerically without further approximations, or it can be solved approximately by using a series expansion for the logarithms. This last method makes it possible to convert the integral equation into a second-order differential equation, and to study dynamical chiral-symmetry breaking analytically. 


\section{A. Differential Equation}

In order to solve the equation analytically we make the replacement $k^{2}+m^{2}(k) \rightarrow$ $k^{2}+m^{2}(0)$, which is a good approximation both for small momenta (where $m(k)$ is almost constant), and for relatively large momenta (where both $m(k)^{2}$ and $m(0)^{2}$ are negligible w.r.t. $\left.k^{2}\right)$. One can also show that this replacement is in agreement with bifurcation analysis, see e.g. 37,25. For very large momenta $(k>\alpha)$ we neglect $m^{2}$ with respect to $k^{2}$ in the denominator completely.

Another approximation, which is commonly made [2], uses the fact that the integral is heavily damped for momenta larger than the mass scale $\alpha$. All the essential physics comes from the infrared part of the nonlinear interal equation. Therefore one uses a series expansion for the logarithms in Eq. (38) for momenta $p, k \ll \alpha$, and introduce a cutoff at $k=\alpha$. However, we are interested in the behavior of the mass function for momenta $p>\alpha$ as well, especially when we include the CS term. Therefore we will adopt a slightly different approximation, which takes into account the ultraviolet tail of the integral as well.

For this purpose we expand the integration kernel $\mathrm{K}(p, k)$ in powers of $\min (p, k) / \max (p, k)$. To leading order this gives

$$
\mathrm{K}(p, k)=2 \frac{\max (p, k)^{2}+2 \alpha^{2}+\alpha \max (p, k)}{\max (p, k)^{3}(\alpha+\max (p, k))}-\frac{4 \alpha^{2}}{\max (p, k)^{4}} \ln (1+\max (p, k) / \alpha) .
$$

Differentiating Eq. (39) with this kernel leads to a second-order differential equation

$$
f(p) m^{\prime \prime}(p)+g(p) m^{\prime}(p)=\frac{4 \alpha}{\pi^{2} N} \frac{p^{2} m(p)}{p^{2}+m^{2}(0)},
$$

with

$$
\begin{aligned}
& f(p)=\frac{1}{K^{\prime}(p, 0)}, \\
& g(p)=\frac{-K^{\prime \prime}(p, 0)}{\left(K^{\prime}(p, 0)\right)^{2}},
\end{aligned}
$$

and two boundary conditions, infrared and ultraviolet ones, just as in the usual approximation scheme. It is easy to show that the behavior of this differential equation in the infrared region is exactly the same as one would obtain by expanding $f$ and $g$ for small momenta directly.

For momenta $p \gg \alpha$ we expand the functions $f(p)$ and $g(p)$, which gives to leading order in $p$

$$
p^{3} m^{\prime \prime}(p)+3 p^{2} m^{\prime}(p)+\frac{16 \alpha}{\pi^{2} N} m(p)=0 .
$$

To solve this we substitute a solution

$$
m(p)=p^{a} \sum_{i=0}^{\infty} c_{i} p^{-i}
$$

and again taking into account leading order only we find $a=0$ or $a=-2$. It is easy to see that $a=0$ is the solution corresponding to explicit chiral-symmetry breaking, since 
this leads to $m(p) \rightarrow m_{0} \neq 0$ in the ultraviolet region. Without a bare mass, the correct solution is $a=-2$, which shows that the dynamical mass function falls off very rapidly in the ultraviolet region. This is also consistent with the ultraviolet boundary condition, but it does not provide a normalization condition.

With this knowledge we now consider integral equation in the infrared region, without neglecting the ultraviolet tail of the integral: we use

$$
m(p)=\alpha^{2} \frac{m(\alpha)}{p^{2}}
$$

for $p>\alpha$, based on the ultraviolet behavior we have just found, and normalized in such a way that the solution is continuous at $p=\alpha$ (we do not require the continuity of derivative, $m^{\prime}(p)$, at $\left.p=\alpha\right)$. Using the usual approximation [2] for $p<\alpha$, we arrive at the integral equation

$$
m(p)=\frac{32}{3 \pi^{2} N} \int_{0}^{\alpha} \mathrm{d} k \frac{k^{2} m(k)}{k^{2}+m^{2}(0)} \frac{1}{\max (k, p)}+\frac{8 \alpha}{\pi^{2} N} \int_{\alpha}^{\infty} \mathrm{d} k \frac{m(k)}{k^{2}},
$$

which finally reduces to

$$
m(p)=\frac{32}{3 \pi^{2} N} \int_{0}^{\alpha} \mathrm{d} k \frac{k^{2} m(k)}{k^{2}+m^{2}(0)} \frac{1}{\max (k, p)}+\frac{8 m(\alpha)}{3 \pi^{2} N} .
$$

This leads to exactly the same second-order differential equation in the infrared region

$$
p^{2} m^{\prime \prime}(p)+2 p m^{\prime}(p)+\frac{32}{3 \pi^{2} N} \frac{p^{2} m(p)}{p^{2}+m^{2}(0)}=0,
$$

as that obtained by neglecting the ultraviolet tail completely, with also the same infrared boundary condition

$$
m^{\prime}(0)=0
$$

The general solution of Eq. (49), satisfying the infrared boundary condition, is given by

$$
m(p)=m(0)_{2} F_{1}\left(a_{+}, a_{-}, \frac{3}{2},-p^{2} / m(0)^{2}\right),
$$

with $a_{ \pm}=\frac{1}{4} \pm \frac{1}{4} i \sqrt{N_{c} / N-1}$ and a critical number of fermion flavors

$$
N_{c}=\frac{128}{3 \pi^{2}}
$$

above which there is no chiral-symmetry breaking. The only difference is the "ultraviolet" boundary condition at $p=\alpha$. This boundary condition now becomes

$$
m(\alpha)+\alpha m^{\prime}(\alpha)=\frac{8 m(\alpha)}{3 \pi^{2} N}
$$

due to the inclusion of the ultraviolet tail of the integral. This boundary condition leads to a slightly different normalization of the infrared mass $m(0)$ (see appendix B for more details) 


$$
\frac{m(0)}{\alpha}=\exp \frac{-2 \pi+2 \phi}{\sqrt{N_{c} / N-1}}
$$

with

$$
\phi=\arg \left[\Gamma\left(1+\frac{i}{2} \sqrt{N_{c} / N-1}\right) \Gamma\left(a_{-}\right)^{2}\left(1-\frac{1}{2} a_{-}\right)\right] .
$$

Close to the critical number of flavors this can be expanded to give

$$
m(0) / \alpha=\exp \left[\frac{-2 \pi}{\sqrt{N_{c} / N-1}}+3 \ln 2+\frac{1}{2} \pi+\frac{2}{7}\right]
$$

which is almost the same as if one neglects the ultraviolet tail, in which case the term $\frac{2}{7}$ in the exponent is absent.

However, this is not the only solution for the boundary condition; it is known that there are infinitely many solutions if $N<N_{c}$ which behave in general as

$$
m(0) / \alpha=\exp \left[\frac{-2 n \pi}{\sqrt{N_{c} / N-1}}+3 \ln 2+\frac{1}{2} \pi+\frac{2}{7}\right],
$$

close to the critical number of fermion flavors. The solution with the largest value of $m / \alpha<1$ corresponds to the ground state, since this has the lowest energy. That means $n=1$ in the above equation, and one can also show that the other solutions have an oscillating behavior at large momenta; only the solution with $n=1$ is a nodeless solution.

\section{B. Integral Equation}

Alternatively, we can solve the nonlinear integral equation for the mass function, Eq. (39), numerically, without further approximations. The integral can be calculated numerically without any cutoff, since the integrand falls off rapidly for large momenta and the integral is finite. Solving the integral equation iteratively leads to qualitatively the same result as the previous analysis.

There is a critical number of fermion flavors below which there is a chiral-symmetrybreaking solution. The critical number is the same as we have just found analytically

$$
N_{c}=\frac{128}{3 \pi^{2}}=4.32
$$

and also the behavior of the infrared mass $m(0)$ is similar as before. In Fig. 1 we have

plotted the infrared mass, or actually $\sqrt{N_{c} / N-1} \ln (m(0) / \alpha)$, versus $\sqrt{N_{c} / N-1}$ for the full integral equation together with the analytical result. We can see that in both cases the mass behaves like

$$
m(0) / \alpha=\exp \left[\frac{-2 \sigma_{1}}{\sqrt{N_{c} / N-1}}+\sigma_{2}\right]
$$


the only difference is the value of the constants $\sigma_{i}$. The analytical result is $\sigma_{1}=\pi$ and $\sigma_{2}=3.94$, whereas the full nonlinear equation gives $\sigma_{1}=3.1$ and $\sigma_{2}=1.8$. So the main difference between the analytical solution of the approximated equation and the numerical solution of the full equation is an overall normalization factor. This difference is due to the fact that in the first case we have made several approximations, but these approximations turn out not to be essential for the behavior of the infrared mass near the critical point. Both in the infrared and in the ultraviolet region the numerical and analytical solutions have the same behavior, see Fig. 2. Of course, these approximations do make a difference in the region where $p$ is of order $\alpha$, but this is to be expected since we expand to leading order only in $p / \alpha$ and $\alpha / p$ respectively.

\section{Chiral Condensate}

The order parameter of the chiral phase transition is the chiral condensate

$$
\langle\bar{\psi} \psi\rangle=\frac{2}{\pi^{2}} \int_{0}^{\infty} \mathrm{d} k \frac{k^{2} m(k)}{k^{2}+m^{2}(k)} .
$$

In order to get an explicit analytical formula for the condensate, we can use Eq. (48), which allows us to write the condensate in terms of the mass function at $p=\alpha$. Using the same linearization as before we get

$$
\langle\bar{\psi} \psi\rangle=\frac{2}{\pi^{2}}\left(\frac{3}{4}+\frac{3 \pi^{2} N}{32}\right) \alpha m(\alpha) .
$$

From this equation we can calculate how the chiral condensate behaves close to the critical number of fermion flavors, see appendix B,

$$
\frac{\langle\bar{\psi} \psi\rangle}{\alpha^{2}}=\frac{608}{7 \pi^{2}} \frac{\Gamma(1 / 2)}{\Gamma(1 / 4)^{2}} \exp \left[\frac{-3 \pi}{\sqrt{N_{c} / N-1}}+\frac{9}{2} \ln 2+\frac{3}{4} \pi+\frac{3}{7}\right] .
$$

Alternatively, we can calculate the condensate numerically, using the numerical solution of the full nonlinear integral equation, which shows a similar behavior, see Fig. 11.

\section{EXPLICIT CHERN-SIMONS TERM}

In this section we add a CS term for the gauge field to the Lagrangian. This breaks the parity explicitly, and gives rise to a parity odd mass term for the fermions, as well as a parity odd part of the gauge boson propagator. We use the gauge boson propagator as discussed in section ППВ, with the inclusion of the leading-order vacuum polarization.

Again we will use a nonlocal gauge-fixing term. For the momentum-dependent gauge function we use [16]

$$
\begin{aligned}
a(q)= & 2 q^{2} D^{T}(q)+2\left(\frac{2 \alpha}{|q|}-1+\frac{4 \alpha \theta}{q^{2}} \arctan \frac{\theta|q|}{\alpha^{2}+\alpha|q|+\theta^{2}}\right. \\
& \left.+\frac{\alpha^{2}-\theta^{2}}{q^{2}} \ln \frac{\alpha^{2}+\theta^{2}}{(\alpha+|q|)^{2}+\theta^{2}}\right)
\end{aligned}
$$


which leads to a wave-function renormalization almost equal to one, the deviation of one is proportional to $\theta B_{ \pm}$, which can be kept very small.

\section{A. Schwinger-Dyson Equation}

With the above gauge boson propagator and gauge function, the SD equation can be rewritten into two decoupled sets of two coupled integral equations for $A_{+}$and $B_{+}$, respectively for $A_{-}$and $B_{-}$. This leads to the following set of equations:

$$
\begin{aligned}
A_{ \pm}(p)= & 1 \pm \frac{8 \alpha}{N p^{2}} \int \frac{\mathrm{d}^{3} k}{(2 \pi)^{3}} \frac{2 B_{ \pm}(k)}{k^{2} A_{ \pm}^{2}(k)+B_{ \pm}^{2}(k)} D^{O}(q) \frac{p \cdot q}{|q|} \\
B_{ \pm}(p)= & \frac{8 \alpha}{N} \int \frac{\mathrm{d}^{3} k}{(2 \pi)^{3}} \frac{1}{k^{2} A_{ \pm}^{2}(k)+B_{ \pm}^{2}(k)} \times \\
& \left(B_{ \pm}(k)\left(2 D^{T}(q)+\frac{a(q)}{q^{2}}\right) \mp 2 A_{ \pm}(k) D^{O}(q) \frac{k \cdot q}{|q|}\right) .
\end{aligned}
$$

The even and odd parts of the scalar function $B(p)$ are

$$
B_{e}(p)=\left(B_{+}(p)+B_{-}(p)\right) / 2,
$$

and

$$
B_{o}(p)=\left(B_{+}(p)-B_{-}(p)\right) / 2,
$$

in terms of $B_{ \pm}(p)$, and similar for $A(p)$. In analyzing these equations it is important to observe that once we have found a solution for $A_{+}(p)$ and $B_{+}(p)$, we automatically have also a solution for $A_{-}(p)$ and $B_{-}(p)$ : namely the set $A_{-}(p)=A_{+}(p)$ and $B_{-}(p)=-B_{+}(p)$. That means that we can always construct a chirally symmetric (but parity odd) solution of the SD equation, with $B_{e}(p)=0$. The question of dynamical chiral-symmetry breaking turns into the question whether or not there exist two (or more) solutions of the set of integral equations.

Without the CS term there is dynamical chiral-symmetry breaking only for $N<N_{c}=$ $128 /\left(3 \pi^{2}\right)$ as we have just seen. We therefore expect a similar situation in the presence of the CS term, at least if the parameter $\theta$ is small. That means that for $N>N_{c}$ we have only the chirally symmetric solution of the above equations, but for $N<N_{c}$ we expect that there are (at least) two solutions for both $B_{+}$and $B_{-}$possible, which can be distinguished by their behavior under the chiral and parity transformations and by their behavior in the limit $\theta \downarrow 0$.

It is also essential to note that in the presence of an explicit CS term for the gauge field in the Lagrangian there is no trivial solution $B=0$, as there would be without the explicit CS term. Due to the explicit breaking of parity, the fermions always acquire a parity-odd mass $B_{o}$, even if the explicit odd mass $m_{o}$ is zero. At large momenta the CS term in the $\mathrm{SD}$ equation dominates (at least if there is no explicit mass term present), which leads to an ultraviolet behavior of the mass functions

$$
B_{+}(p) \sim-B_{-}(p) \sim \frac{\theta}{p},
$$


at a perturbative level, whereas in the absence of the CS term the mass function $m(p)$ falls off like $1 / p^{2}$, as we have seen in the previous section. We will discuss this point in more detail when we are studying the breakdown of chiral symmetry analytically.

\section{B. Numerical Results}

We have first solved the set of coupled integral equations Eq. (64) and (65) numerically without further approximations. Depending on the values of the number of fermion flavors $N$ and the CS coefficient $\theta$, there is only one (chirally symmetric) solution in most parameter space, but we found two solutions, allowing for a chiral-symmetry-breaking solution, for small $\theta$ and small $N$, in agreement with the above expectations. Using the notation $B_{ \pm}(p)$ and $\tilde{B}_{ \pm}(p)$ for the two different solutions of the above equations, we have found the following solutions:

1. $B_{+}(0)=-B_{-}(0)=\mathcal{O}(m(0))>0$, with $B_{+}(p)=-B_{-}(p)>m(p)$, $B_{e}(p) \equiv 0$ and $B_{o}(0)=\mathcal{O}(m(0))$

for $N<N_{c}(\theta=0)$ we find that $B_{ \pm}(p)$ and thus $B_{o}(p)$ go towards the nontrivial solution $m(p)$ in the limit $\theta \downarrow 0$;

for $N>N_{c}(\theta=0)$ we find that $B_{+}(p)=B_{o}(p)=\mathcal{O}(\theta)$, which vanishes in the limit $\theta \downarrow 0$.

2. $B_{+}(0) \sim \tilde{B}_{-}(0)=\mathcal{O}(m(0))$, with $B_{+}(p)>m(p)>\tilde{B}_{-}(p)$

$B_{e}(p)=\mathcal{O}(m(p))$ and $B_{o}(p)=\mathcal{O}(\theta)$

This solution exists for values of $N<N_{c}(\theta=0)$ and small (compared to $\alpha$ ) values of $\theta$ only. There is a critical number $N_{c}(\theta)<N_{c}(0)$ for given $\theta$, or critical $\theta_{c}(N)$ for given $N<N_{c}(0)$. In the limit $\theta \downarrow 0$ both $B_{ \pm}$and thus $B_{e}$ go towards the nontrivial solution $m(p): B_{+}(p) \downarrow m(p)$ and $\tilde{B}_{-}(p) \uparrow m(p)$ in this limit. The parity breaking solution $B_{o}(p)$ vanishes in this limit.

3. $\tilde{B}_{+}(0)=-\tilde{B}_{-}(0)=-\mathcal{O}(m(0))<0$

The chirally symmetric combination, which exists for small values of $N$ and small values of $\theta$ only.

4. $\tilde{B}_{+}(0) \sim B_{-}(0)=-\mathcal{O}(m(0))$

The fourth possible combination of $B_{+}$and $B_{-}$, also existing for small values of $N$ and small values of $\theta$ only.

The typical behavior of the numerical solutions for $A$ and $B$ is shown in Fig. 3. As expected, $A_{ \pm}$is indeed very close to one, due to our choice of the gauge function. The solution $B_{ \pm}(p)$ exists for all values of both $N$ and $\theta$, allowing only a chirally symmetric solution. The other solution, $\tilde{B}_{ \pm}(p)$, is the interesting one, leading to dynamical chiralsymmetry breaking. The iterative process of solving the integral equation numerically does not converge to a (second) stable solution $\tilde{B}_{ \pm}$for all values of $N$ and $\theta$ : we can find this solution only for $N$ below some critical value (depending on $\theta$ ) and $\theta$ below some critical value (depending on $N$ ), thus showing a chiral phase transition at some critical $\theta$ and $N$.

Our numerical results all indicate strongly that this phase transition is a first-order phase transition, in contrast to the infinite-order phase transition in pure QED $(\theta=0)$, although it 
is numerically very difficult to establish the type of phase transition at the critical values of $N$ and $\theta$. For fixed $\theta$ and small $N$, there exists a second solution, $\tilde{B}_{ \pm}(p)$, as can be seen from Fig. 4. The value at the origin $\tilde{B}_{-}(0)$ decreases rapidly for increasing $N$ and this solution "disappears" (without $B_{ \pm}(0)$ going to zero) at some critical value $N<N_{c}(\theta=0)$, and also $B_{e}(0)$ does not go to zero at this critical value. Considering $B_{e}(0)$ as the order parameter for the chiral phase transition, this corresponds to a first-order phase transition, in contrast to the infinite-order phase transition at $\theta=0$.

If we look at the behavior of $\tilde{B}_{-}(0)$ and $B_{e}(0)$ at fixed $N<N_{c}(\theta=0)$ and increase $\theta$, we see a similar situation. For (very) small values of $\theta$ we find the two solutions $B$ and $\tilde{B}$ leading to chiral-symmetry breaking. For increasing $\theta$ we find that $\tilde{B}(0)$ decreases, and disappears at some critical value $\theta_{c}(N)$, without going to zero at this critical value, see Fig. 5 .

\section{Condensate}

Although both functions $B_{ \pm}(p)$ behave like $\theta / p$ in the ultraviolet region, leading to divergent integrals for the condensates $\langle\bar{\psi} \psi\rangle_{ \pm}$, the chiral condensate is convergent due to the fact that the leading-order contributions in $B_{+}(p)$ and $B_{-}(p)$ (or $\left.\tilde{B}_{-}(p)\right)$ cancel. So the chiral order parameter is

$$
\langle\bar{\psi} \psi\rangle=\frac{1}{\pi^{2}} \int_{0}^{\infty} \mathrm{d} k\left(\frac{k^{2} B_{+}(k)}{A_{+}^{2}(k) k^{2}+B_{+}^{2}(k)}+\frac{k^{2} \tilde{B}_{-}(k)}{\tilde{A}_{-}^{2}(k) k^{2}+\tilde{B}_{-}^{2}(k)}\right) .
$$

The chirally symmetric combination, $\left(B_{+}(p), B_{-}(p)\right)$ with $B_{e}=0$, gives $\langle\bar{\psi} \psi\rangle=0$, as would be expected from a chirally symmetric solution. Note that the other condensate, $\langle\bar{\psi} \tau \psi\rangle$, is actually logarithmic divergent because the leading-order contributions add up. Once we have the numerical solutions, it is straightforward to calculate this chiral condensate as well, see Fig. 5. The behavior of the chiral condensate also indicates a first-order phase transition.

However, as we mentioned before, it is numerically very difficult to establish such a first-order phase transition. In order to confirm that it is indeed a first-order transition and to determine the critical parameters $N_{c}$ and $\theta_{c}$, we have to study the phase transition analytically. We can do this by solving the integral equations analytically, after some further approximations analogously to the approximations leading to analytic solution in the pure QED case.

\section{ANALYTICAL STUDY}

In order to see whether there is indeed a first-order phase transition, we have analyzed the SD equation analytically, after some more approximations. Based on the fact that $A(p) \equiv 1$ exactly if $\theta$ is zero, and very close to one for small values of $\theta$ (small compared to $\alpha$ ), we replace $A(p)$ by one, so we get the following equation for $B_{ \pm}$

$$
\begin{aligned}
B_{ \pm}(p)= & \frac{8 \alpha}{N} \int \frac{\mathrm{d}^{3} k}{(2 \pi)^{3}} \frac{1}{k^{2}+B_{ \pm}^{2}(k)} \times \\
& \left(B_{ \pm}(q)\left(2 D^{T}(q)+\frac{a(q)}{q^{2}}\right) \mp 2 D^{O}(q) \frac{k \cdot q}{|q|}\right) .
\end{aligned}
$$


The error we make in neglecting the effects of the wave-function renormalization (even if we use the nonlocal gauge) is of the order of $\theta B_{ \pm}$, which is (close to the critical number of fermion flavors in the absence of the CS term) of the order of $\theta^{2}$, because the parity odd mass, generated by the CS term, is of order $\theta$. So for a consistent approximation in order to get an analytical solution of the equation, it is enough to expand the kernel in $\theta$ and neglect all terms of order $\theta^{2}$ and higher. 3 Expanding the nonlocal gauge in $\theta$ gives

$$
a(q)=\frac{2 q}{\alpha+q}+\frac{4 \alpha}{q}-2-\frac{4 \alpha^{2}}{q^{2}} \ln \left(\frac{\alpha+q}{\alpha}\right)+\mathcal{O}\left(\theta^{2}\right) .
$$

So to order $\theta$ it is the same as the nonlocal gauge without the CS term, see Eq. (37). By inspection of the transverse part $D^{T}\left(q^{2}\right)$ of the photon propagator it is easy to see that up to order $\theta$ this is also exactly the same as without the CS term. That means that the second term on the RHS of Eq. (70), proportional to $D^{O}(q)$, is the only $\mathcal{O}(\theta)$ contribution in the SD equation for $B_{ \pm}$, and that for the other terms we can use the same kind of approximations as in section III] on pure QED.

So in order to study the problem of dynamical chiral-symmetry breaking analytically, we replace $k^{2}+B_{ \pm}^{2}(p)$ by $k^{2}+M_{ \pm}^{2}$, where $M_{ \pm} \equiv B_{ \pm}(0)$, in the denominator of the integrand. In general we get

$$
\begin{aligned}
B_{ \pm}(p)= & \frac{32}{3 \pi^{2} N} \int_{0}^{\infty} \mathrm{d} k \frac{k^{2} B_{ \pm}(k)}{k^{2}+M_{ \pm}^{2}} K(p, k) \\
& \pm \frac{4 \alpha \theta}{\pi^{2} N} \int_{0}^{\infty} \frac{k^{2} \mathrm{~d} k}{k^{2}+M_{ \pm}^{2}} \int_{-1}^{1} \mathrm{~d} z \frac{k \cdot(k-p)}{(k-p)^{2}(|k-p|+\alpha)^{2}}
\end{aligned}
$$

with the kernel given by Eq. (38), and $z=\cos \phi$, with the angle $\phi$ between the vectors $p$ and $k: p \cdot k=p k \cos \phi$. For momenta $p<\alpha$ this reduces to

$$
\begin{aligned}
B_{ \pm}(p)= & \frac{32}{3 \pi^{2} N} \int_{0}^{\alpha} \mathrm{d} k \frac{k^{2}}{k^{2}+M_{ \pm}^{2}} \frac{B_{ \pm}(k)}{\max (p, k)}+\frac{8 \alpha}{\pi^{2} N} \int_{\alpha}^{\infty} \mathrm{d} k \frac{B_{ \pm}(k)}{k^{2}} \\
& \pm \frac{4 \alpha \theta}{\pi^{2} N} \int_{0}^{\infty} \frac{k^{2} \mathrm{~d} k}{k^{2}+M_{ \pm}^{2}} \int_{-1}^{1} \mathrm{~d} z \frac{k \cdot(k-p)}{(k-p)^{2}(|k-p|+\alpha)^{2}}
\end{aligned}
$$

In order to get this equation, we have used the same approximations as in section III for the first term of the integral, which is independent of $\theta$.

\section{A. Explicit Chern-Simons Contribution}

The last term on the RHS in Eq. (73), proportional to $\theta$,

$$
I_{\theta}(p) \equiv \frac{4 \alpha \theta}{\pi^{2} N} \int_{0}^{\infty} \frac{k^{2} \mathrm{~d} k}{k^{2}+M_{ \pm}^{2}} \int_{-1}^{1} \mathrm{~d} z \frac{k \cdot(k-p)}{(k-p)^{2}(|k-p|+\alpha)^{2}}
$$

\footnotetext{
${ }^{3}$ This should be compared with the analysis by Hong and Park 40 where the order $\theta$ term was neglected from the beginning.
} 
can be calculated analytically, see appendix A. For the radial integration we expand the integration kernel for $p<q$ and $p>q$ and take into account only the leading-order terms in $\min (p, q) / \max (p, q)$. Furthermore, we neglect $M_{ \pm}$with respect to $\alpha$, which is justified close to the critical number of fermion flavors and for small values of $\theta$. So in the far infrared region the leading contribution coming from the explicit CS term behaves like

$$
I_{\theta}(p)=\frac{8}{\pi^{2} N} \theta+\mathcal{O}(p)
$$

and in the far ultraviolet region the leading-order behavior is

$$
I_{\theta}(p)=\frac{32}{3 \pi^{2} N} \frac{\alpha}{p} \theta+\mathcal{O}\left(1 / p^{2}\right) .
$$

This means that in the ultraviolet region the CS term will dominate, since we know that the mass function $B(p)$ in the absence of the CS term behaves like $1 / p^{2}$ in the far ultraviolet. Higher order contributions in $\min (p, q) / \max (p, q)$ will change this result only quantitatively, but not affect the general behavior.

Based on these expansions, we use an analytical (continuous) approximation for the contribution from the $\theta$ term

$$
I_{\theta}(p)= \begin{cases}\frac{8 \theta}{\pi^{2} N} & \text { for } p<\alpha \\ \frac{32 \theta}{3 \pi^{2} N}\left(\frac{\alpha}{p}-\frac{\alpha^{2}}{4 p^{2}}\right) & \text { for } p>\alpha\end{cases}
$$

for small values of $\theta$ compared to $\alpha$.

\section{B. Ultraviolet Behavior}

The next thing we have to calculate before we solve the integral equation in the infrared regoin, is the ultraviolet tail

$$
\frac{8 \alpha}{\pi^{2} N} \int_{\alpha}^{\infty} \mathrm{d} k \frac{B_{ \pm}(k)}{k^{2}}
$$

but for this purpose we have to know the ultraviolet behavior of the mass function. From the integral equation we can derive a second-order differential equation for momenta $p \gg \alpha$

$$
f(p)\left(B_{ \pm}^{\prime \prime}(p) \mp I_{\theta}^{\prime \prime}(p)\right)+g(p)\left(B_{ \pm}^{\prime}(p) \mp I_{\theta}^{\prime}(p)\right)=\frac{4 \alpha}{\pi^{2} N} B_{ \pm}(p),
$$

with $f(p)$ and $g(p)$ given by Eq. (42) and (43) respectively. In the ultraviolet region, these functions behave like

$$
\begin{aligned}
f(p) & =\frac{-p^{3}}{4}, & g(p) & =\frac{-3 p^{2}}{4}, \\
I_{\theta}^{\prime}(p) & =\frac{-32 \theta \alpha}{3 \pi^{2} N p^{2}}, & I_{\theta}^{\prime \prime}(p) & =\frac{64 \theta \alpha}{3 \pi^{2} N p^{3}} .
\end{aligned}
$$

The leading ultraviolet behavior of the solution of this equation is either constant (which would correspond to an explicit mass term in the original integral equation), or 


$$
B_{ \pm}(p)= \pm I_{\theta}(p)=\frac{32}{3 \pi^{2} N} \frac{\alpha}{p} \theta
$$

which is the correct solution in this case, and also in agreement with the ultraviolet boundary condition. This is the same as the perturbatively dominant behavior, as could also be seen directly from the original integral equation, assuming that the mass function falls off rapidly enough in the ultraviolet region. In order to have a continuous solution at $p=\alpha$, we use the next-to-leading order term, so we have

$$
B_{ \pm}(p)= \pm \frac{32}{3 \pi^{2} N} \frac{\alpha}{p} \theta+\frac{\alpha^{2}}{p^{2}}\left(B_{ \pm}(\alpha) \mp \frac{32 \theta}{3 \pi^{2} N}\right)
$$

for $p>\alpha$.

\section{Analytical Results}

So we arrive at the integral equation for momenta $p<\alpha$

$$
B_{ \pm}(p)=\frac{32}{3 \pi^{2} N} \int_{0}^{\alpha} \mathrm{d} k \frac{k^{2}}{k^{2}+M_{ \pm}^{2}} \frac{B_{ \pm}(k)}{\max (p, k)}+\frac{8}{3 \pi^{2} N}\left( \pm \frac{16 \theta}{3 \pi^{2} N}+B_{ \pm}(\alpha)\right) \pm \frac{8 \theta}{\pi^{2} N}
$$

which we can now solve analytically by converting it to a second-order differential equation with boundary conditions.

The second-order differential equation is the same as without the CS term, Eq. (49), and also the infrared boundary condition is the same, the only (but essential!) difference is the "ultraviolet" boundary condition at $p=\alpha$

$$
B_{ \pm}(\alpha)+\alpha B_{ \pm}^{\prime}(\alpha)=\frac{8}{3 \pi^{2} N}\left( \pm \frac{16 \theta}{3 \pi^{2} N}+B_{ \pm}(\alpha)\right) \pm \frac{8 \theta}{\pi^{2} N}
$$

which should be compared with Eq. (53). It is also important to keep in mind that the normalization condition is

$$
B_{ \pm}(0)=M_{ \pm}
$$

and that the SD equation does determine the sign of the mass function as well. This is obviously not the case if $\theta=0$.

The general solution of the differential equation satisfying the infrared boundary condition and the normalization condition is

$$
B_{ \pm}(p)=M_{ \pm 2} F_{1}\left(a_{+}, a_{-}, \frac{3}{2} ;-p^{2} / M_{ \pm}^{2}\right)
$$

where $a_{ \pm}=\frac{1}{4}\left(1 \pm i \sqrt{N_{c} / N-1}\right)$. The ultraviolet boundary condition leads to the condition

$$
\begin{array}{r}
M_{ \pm 2} F_{1}\left(a_{+}, a_{-}, \frac{1}{2} ;-\alpha^{2} / M_{ \pm}^{2}\right)-\frac{8}{3 \pi^{2} N} M_{ \pm 2} F_{1}\left(a_{+}, a_{-}, \frac{3}{2} ;-\alpha^{2} / M_{ \pm}^{2}\right) \\
= \pm \frac{8 \theta}{\pi^{2} N}\left(1+\frac{16}{9 \pi^{2} N}\right) .
\end{array}
$$


In order to determine $M$, we can plot the LHS of the above equation divided by $\frac{8}{\pi^{2} N}\left(1+\frac{16}{9 \pi^{2} N}\right)$ as function of $M$ for a given value of $N$, which gives us automatically $\theta$ as function of $M$, see Fig. 6a. From this figure we can see that there are three solutions possible for $B_{+}$and $B_{-}$. Two of them correspond to the two solutions which we have found numerically, and the third is an oscillating solution which in the limit of vanishing $\theta$ corresponds to the first oscillating solution in pure QED, $n=2$ in Eq. (57). For extremely small values of $M$ and $\theta$ there are more oscillating solutions, in the region around the origin, but these are numerically unstable, and correspond to higher excites states, just as in pure QED.

Using the same notation as for the numerical solution, we have $B_{+}(0)>0$ and $\tilde{B}_{+}(0)<0$, and for $B_{-}$the opposite signs. The set $\left(B_{+}, B_{-}\right)$is the chirally symmetric solution which is present for all values of $(N, \theta)$. The chiral-symmetry-breaking solution is given by the set $\left(B_{+}, \tilde{B}_{-}\right)$(or vice versa, the set $\left.\left(\tilde{B}_{+}, B_{-}\right)\right)$. In the limit $\theta \rightarrow 0$, this chiral-symmetry-breaking solution behaves in the following way

$$
\begin{aligned}
& B_{e} \rightarrow m, \\
& B_{o}=\mathcal{O}(\theta) .
\end{aligned}
$$

If we increase $\theta$ from zero at fixed $N$, then the absolute value of the second solution, $\tilde{B}_{ \pm}$, decreases (whereas the other solution increases), until at a critical value $\theta_{c}$ this solution coincides with a third solution, and disappears, but does not become zero at the critical point, see Fig. 6la. This clearly signals a first-order phase transition, as was also suggested by the numerical results. For comparison, we also showed our numerical data in the same figure, where we use a different value of $\alpha$ for our numerical and analytical calculation, $\alpha_{\text {num }}$ and $\alpha_{\text {an }}$ respectively, in order to have equal numerical and analytical values of $m(0) / \alpha_{\text {num }}$ in the absence of the CS term (note that the axes are $\theta / \alpha_{\text {num }}$ and $|B(0)| / \alpha_{\text {num }}$ respectively). This shows clearly that both our numerical and our analytical results are in good agreement with each other.

We can also plot $M$ versus $N$ for some fixed values of $\theta$, see Fig. 6b. From that figure we can see that if we increase $N$ for fixed $\theta$, the chiral-symmetry-breaking solutions disappear if $N$ exceeds some critical value $N_{c}$, which decreases rapidly as a function of $\theta$. This figure shows that the chiral phase transition is first order in this direction as well: increasing $N$ beyond $N_{c}(\theta)$ makes the second (and third) solution disappear, but at the phase transition neither $\tilde{B}$ nor $B_{e}=\left(B_{+}+B_{-}\right) / 2$ (which can be regarded as the order parameter of the chiral phase transition) becomes zero. In this figure we can also see that in the limit $\theta \rightarrow 0$ the critical value $N_{c}$ goes towards $N_{c}(0) \simeq 4.32$.

The critical parameters $N_{c}$ and $\theta_{c}$ can be calculated by differentiating the ultraviolet boundary condition, Eq. (86), with respect to $M$. This leads to an equation for $M_{c}$ as function of $N_{c}$ (remember that $M$ does not vanish at $N_{c}$ )

$$
\begin{aligned}
{ }_{2} F_{1}\left(a_{+}, a_{-}, \frac{-1}{2} ;-\alpha^{2} / M_{c}^{2}\right) & +\frac{8}{3 \pi^{2} N}{ }_{2} F_{1}\left(a_{+}, a_{-}, \frac{1}{2} ;-\alpha^{2} / M_{c}^{2}\right) \\
& -\frac{16}{3 \pi^{2} N}{ }_{2} F_{1}\left(a_{+}, a_{-}, \frac{3}{2} ;-\alpha^{2} / M_{c}^{2}\right)=0,
\end{aligned}
$$

which can be used as input for the ultraviolet boundary condition itself in order to calculate $\theta_{c}$. Although we do not have an explicit form for $\theta_{c}$ as function of $N$, we can calculate it 
numerically, and have shown the critical line in Fig. 7. In this figure we also show some estimates of the critical parameters based on our numerical calculation. This shows that the numerical and analytical results are qualitatively in good agreement with each other, and the only difference is an overall scale factor (just as in pure QED).

For small values of $M_{c}$ and $N_{c}(\theta)$ close to $N_{c}(0) \simeq 4.32$, we can expand the above equation in $\sqrt{N_{c}(0) / N-1}$, leading to

$$
M_{c} / \alpha=\exp \left[\frac{-2 \pi}{\sqrt{N_{c}(0) / N-1}}+\frac{1}{2} \pi+3 \ln 2-\frac{8}{21}\right]
$$

(see appendix B), which can be inserted into the series expansion for $\theta$. To leading order in $\sqrt{N_{c}(0) / N-1}$, this gives the following expression for $\theta_{c}$

$$
\theta_{c} \simeq \frac{448 \Gamma(1 / 2)}{75 \Gamma(1 / 4)^{2}} \exp \left[\frac{-3 \pi}{\sqrt{N_{c} / N-1}}+\frac{3}{4} \pi+\frac{9}{2} \ln 2-\frac{4}{7}\right] .
$$

As mentioned before, we can also calculate the chiral condensate which is well-defined, even without cutoff. The chiral condensate is

$$
\langle\bar{\psi} \psi\rangle=\frac{1}{\pi^{2}} \int_{0}^{\infty} \mathrm{d} k\left(\frac{k^{2} B_{+}(k)}{A_{+}^{2}(k) k^{2}+B_{+}^{2}(k)}+\frac{k^{2} \tilde{B}_{-}(k)}{\tilde{A}_{-}^{2}(k) k^{2}+\tilde{B}_{-}^{2}(k)}\right) .
$$

In the approximations we are using here, this reduces to

$$
\langle\bar{\psi} \psi\rangle=\frac{1}{\pi^{2}} \int_{0}^{\alpha} \mathrm{d} k\left(\frac{k^{2} B_{+}(k)}{k^{2}+M_{+}^{2}}+\frac{k^{2} \tilde{B}_{-}(k)}{k^{2}+\tilde{M}_{-}^{2}}\right)+\frac{1}{\pi^{2}} \int_{\alpha}^{\infty} \mathrm{d} k\left(B_{+}(k)+\tilde{B}_{-}(k)\right)
$$

which can be calculated analytically, using Eq. (82) at $p=\alpha$ and the ultraviolet expansion Eq. 81 for $B_{ \pm}$. This leads to

$$
\langle\bar{\psi} \psi\rangle=\frac{\alpha}{\pi^{2}}\left(B_{+}(\alpha)+\tilde{B}_{-}(\alpha)\right)\left(\frac{3 \pi^{2} N}{32}+\frac{3}{4}\right)
$$

with $B_{+}(\alpha)$ and $\tilde{B}_{-}(\alpha)$ determined through Eqs. (85) and (86). In Fig. 8 we show the chiral condensate (together with the $B_{e}(0)$ ) as obtained both numerically and analytically for $\theta=0$ and $\theta=10^{-5}$ as a function of $N$. Again, they are qualitatively in good agreement with each other, and show a discontinuity in both the chiral condensate and $B_{e}(0)$ at the critical point.

In Fig. 9, we show both $B_{e}(p)$ and $B_{o}(p)$, as obtained numerically and analytically. The numerical and the analytical solutions have the same behavior in both the infrared and the ultraviolet region; around $p=\alpha$ there is of course a kink in the analytical solution due to the approximations we have made. There is a scale difference between the analytical and the numerical solution for the chiral-symmetry-breaking solution, just as in pure QED; in fact, if we compare this figure with Fig. 2 we can see that the parity-even mass function is almost the same as the dynamical mass function in pure QED. Furthermore, this figure shows very well the difference in the ultraviolet between the even and the odd mass function: the odd mass function behaves like $\theta / p$, whereas the even mass function $B_{e}(p)=\left(B_{+}(p)+\tilde{B}_{-}(p)\right) / 2$ behaves like $1 / p^{2}$, due to the cancellation of the terms proportional to $\pm \theta / p$. 


\section{CJT EFFECTIVE POTENTIAL}

We consider the effective action of Cornwall-Jackiw-Tomboulis [36] (CJT) which is given by

$$
\Gamma[S]=\Gamma_{0}[S]+\Gamma_{1}[S]
$$

with

$$
\begin{aligned}
& \Gamma_{0}[S] \equiv-\operatorname{Tr}\left[\operatorname{Ln}\left[S^{-1} S_{0}\right]+S_{0}^{-1} S-1\right] \\
& \Gamma_{1}[S] \equiv \frac{e^{2}}{2} \operatorname{Tr}\left[S \gamma_{\mu} D_{\mu \nu} \gamma_{\nu} S\right]
\end{aligned}
$$

where $D_{\mu \nu}$ is the full photon propagator, and $S_{0}$ and $S$ are the bare and the full fermion propagator respectively. At the stationary point $\delta \Gamma[S] / \delta S=0$, this effective action actually gives the SD equation: $S^{-1}-S_{0}^{-1}=-e^{2} \operatorname{Tr}\left[\gamma_{\mu} D_{\mu \nu} \gamma_{\nu} S\right]$, as is shown explicitly in appendix C. Note that we have chosen the bare vertex to write the effective action, which can be justified by taking the nonlocal gauge. Furthermore we remark that the effective action is normalized so that $\Gamma_{0}\left[S=S_{0}\right]=0$. For a review of the effective potential, see e.g. 41.

We can rewrite the above expression in terms of the fermion propagators $S_{+}$and $S_{-}$

$$
S=S_{+} \chi_{+}+S_{-} \chi_{-} \cdot
$$

By using the fact that $\chi_{+}$and $\chi_{-}$are projection operators and the relation $\operatorname{Tr} \operatorname{Ln}=\operatorname{Ln}$ Det, it is not difficult to show that both $\Gamma_{0}[S]$ and $\Gamma_{1}[S]$ are decomposed into two parts

$$
\Gamma_{0,1}[S]=\Gamma_{0,1}\left[S_{+}\right]+\Gamma_{0,1}\left[S_{-}\right]
$$

where

$$
\Gamma_{0}\left[S_{ \pm}\right] \equiv-\operatorname{Tr}\left[\operatorname{Ln}\left[S_{ \pm}^{-1} S_{0}\right]+S_{0}^{-1} S_{ \pm}-1\right]
$$

and

$$
\Gamma_{1}\left[S_{ \pm}\right] \equiv \frac{e^{2}}{2} \int \frac{\mathrm{d}^{3} p}{(2 \pi)^{3}} \int \frac{\mathrm{d}^{3} k}{(2 \pi)^{3}} \operatorname{Tr}\left[\gamma^{\mu} S_{ \pm}(p) \gamma^{\nu} S_{ \pm}(k) \chi_{ \pm}\right] D_{\mu \nu}(k-p)
$$

Now we define the effective potential $V[S]$ by dividing the effective action by the spacetime volume $\int d^{3} x: V[S] \equiv \Gamma[S] / \int d^{3} x$. In momentum space, $V_{0}\left[S_{ \pm}\right]$is given by

$$
\begin{aligned}
V_{0}\left[S_{ \pm}\right]= & \frac{N}{2 \pi^{2}} \int_{0}^{\infty} p^{2} \mathrm{~d} p\left[-\ln \left(\frac{p^{2} A_{ \pm}^{2}(p)+B_{ \pm}^{2}(p)}{p^{2}}\right)\right. \\
& \left.+2 \frac{p^{2} A_{ \pm}(p)\left[A_{ \pm}(p)-1\right]+B_{ \pm}^{2}(p)}{p^{2} A_{ \pm}^{2}(p)+B_{ \pm}^{2}(p)}\right]
\end{aligned}
$$

For $V_{1}\left[S_{ \pm}\right]$, after calculating the trace in the integrand, we arrive at the result 


$$
\begin{aligned}
& V_{1}\left[S_{ \pm}\right]=e^{2} \int \frac{\mathrm{d}^{3} p}{(2 \pi)^{3}} \int \frac{\mathrm{d}^{3} k}{(2 \pi)^{3}} \frac{1}{\left[p^{2} A_{ \pm}^{2}(p)+B_{ \pm}^{2}(p)\right]\left[k^{2} A_{ \pm}^{2}(k)+B_{ \pm}^{2}(k)\right]} \\
& \times\left\{\left(2 D_{T}(q) \frac{(q \cdot p)(q \cdot k)}{q^{2}}+\frac{a(q)}{q^{4}}(2(q \cdot p)(q \cdot k)-p \cdot k)\right) A_{ \pm}(p) A_{ \pm}(k)\right. \\
& \left.-\left(2 D_{T}(q)+\frac{a(q)}{q^{2}}\right) B_{ \pm}(p) B_{ \pm}(k) \pm \frac{D_{O}(q)}{|q|}\left[q \cdot p A_{ \pm}(p) B_{ \pm}(k)-q \cdot k B_{ \pm}(p) A_{ \pm}(k)\right]\right\}
\end{aligned}
$$

with $q=k-p$. At this stage we adopt the nonlocal gauge $a(q)$ which leads to no wavefunction renormalization for the fermion: $A_{ \pm}(p) \equiv 1$. Then we can write

$$
V[B]=\left.V[S]\right|_{A_{ \pm} \equiv 1}=V_{0}\left[B_{+}\right]+V_{0}\left[B_{-}\right]+V_{1}\left[B_{+}\right]+V_{1}\left[B_{-}\right]
$$

where

$$
V_{0}\left[B_{ \pm}\right]=\frac{N}{2 \pi^{2}} \int_{0}^{\infty} p^{2} \mathrm{~d} p\left[-\ln \left(\frac{p^{2}+B_{ \pm}^{2}(p)}{p^{2}}\right)+2 \frac{B_{ \pm}^{2}(p)}{p^{2}+B_{ \pm}^{2}(p)}\right]
$$

and

$$
\begin{aligned}
& V_{1}\left[B_{ \pm}\right]=e^{2} \int \frac{\mathrm{d}^{3} p}{(2 \pi)^{3}} \int \frac{\mathrm{d}^{3} k}{(2 \pi)^{3}} \frac{1}{\left[p^{2}+B_{ \pm}^{2}(p)\right]\left[k^{2}+B_{ \pm}^{2}(k)\right]} \\
& \times\left\{\left(-2 D_{T}(q)-\frac{a(q)}{q^{2}}\right) B_{ \pm}(p) B_{ \pm}(k) \pm \frac{D_{O}(q)}{|q|}\left[(q \cdot p) B_{ \pm}(k)-(q \cdot k) B_{ \pm}(p)\right]\right\} .
\end{aligned}
$$

Actually this reproduces the SD equation for $B_{ \pm}$in the nonlocal gauge at the stationary point $\delta V[B] / \delta B_{ \pm}=0$ as shown in appendix $\mathrm{C}$.

At the stationary point of $V[B], B_{ \pm}$satisfies the $\mathrm{SD}$ equation and hence $B_{ \pm}$is given by the solution to the SD equation: $B_{ \pm}=B_{ \pm}^{\text {sol }}$. Therefore, at the stationary point, we obtain

$$
V_{1}\left[B_{ \pm}\right]=-\int \frac{\mathrm{d}^{3} p}{(2 \pi)^{3}} \frac{B_{ \pm}(p)\left[B_{ \pm}(p)-m_{ \pm}\right]}{p^{2}+B_{ \pm}^{2}(p)} .
$$

Hence the effective potential at the stationary point is given by

$$
\begin{aligned}
V\left[B_{\text {sol }}\right] & =V\left[B_{+}^{\text {sol }}\right]+V\left[B_{-}^{s o l}\right] \\
V\left[B_{ \pm}\right] & =-\int \frac{\mathrm{d}^{3} p}{(2 \pi)^{3}}\left[\ln \left(1+\frac{B_{ \pm}^{2}(p)}{p^{2}}\right)-\frac{B_{ \pm}(p)\left[B_{ \pm}(p)+m_{ \pm}\right]}{p^{2}+B_{ \pm}^{2}(p)}\right] .
\end{aligned}
$$

Note that the function $g(x)=\ln (1+x)-\frac{x}{1+x}$ is positive and monotonically increasing in $x(>0)$. Therefore, when $m_{ \pm}=0$,

$$
V\left[B_{\text {sol }}\right]=-\frac{1}{2 \pi^{2}} \int_{0}^{\infty} p^{2} \mathrm{~d} p\left[g\left(\frac{B_{+}^{2}(p)}{p^{2}}\right)+g\left(\frac{B_{-}^{2}(p)}{p^{2}}\right)\right]
$$

is non-positive, $V\left[B_{\text {sol }}\right] \leq 0$, and all the nontrivial solutions have lower energy than the trivial ones (in the case of pure QED). Therefore we can determine the ground state, namely the solution which minimizes the effective potential, by using Eq. (109). 
Without actually calculating the integral, we can easily conclude that in the presence of an explicit CS term, the chirally symmetric solution $\left(B_{+}, B_{-}\right)$gives a lower effective potential and is thus favored above the chiral-symmetry-breaking solution $\left(B_{+}, \tilde{B}_{-}\right)$. Since the effective potential is the sum of two non-positive integrals, each of which depends on $B_{+}^{2}$ or $B_{-}^{2}$, this effective potential is minimized by a symmetric set of solutions $\left(B_{+}, B_{-}\right)$and not by $\left(B_{+}, \tilde{B}_{-}\right)$. So in the presence of an explicit CS term, the chirally symmetric phase is the ground state, even if the number of fermion flavors $N$ and the CS parameter $\theta$ are below their critical values for dynamical chiral-symmetry breaking. This is quite surprising, since it is well-known that without the explicit CS term the chiral symmetry is broken for $N$ below the critical number, as can also be seen from the effective potential, Eq. (109).

In solving the SD equation we have put the external source $m_{e}$ and $m_{o}$ to zero from the beginning. As a result, the solution for the homogeneous SD equation has no specific direction for the dynamical mass to be generated. Actually, if a solution is found for $B_{+}$, then another solution $B_{-}=-B_{+}$is automatically obtained, and it is this solution which gives the lowest effective potential. In order to study the spontaneous breaking of a symmetry in general, one can introduce an external source $J \mathcal{O}$ which breaks the symmetry in question and subsequently consider the limit of removing the external source. If the symmetry is broken even in this limit, which is signaled by the non-vanishing order parameter $\phi=\lim _{J \rightarrow 0}\langle\mathcal{O}\rangle_{J}$, then it is said that the symmetry is spontaneously broken. In taking this limit one must specify from which direction the external source is decreased to zero, $J \rightarrow 0$.

In this case we must consider the two limits: $m_{e} \rightarrow 0, m_{o} \rightarrow 0$. For simplicity we keep $m_{e}$ and $m_{o}$ positive, $m_{e}, m_{o} \geq 0$ and consider the limit: $m_{e} \downarrow 0, m_{o} \downarrow 0$, without loss of generality. Then there are five cases to be examined, which are given below.

\begin{tabular}{llll}
\multicolumn{2}{c}{ explicit masses } & \multicolumn{1}{c}{ condensates } & \multicolumn{1}{c}{ solutions of SD eq. } \\
\hline$m_{e}>m_{o}>0$ & $m_{+}>m_{-}>0$ & $\langle\bar{\psi} \psi\rangle_{+}>\langle\bar{\psi} \psi\rangle_{-}>0$ & $B_{+}(0)>0, \tilde{B}_{-}(0)>0$ \\
$m_{e}>0, m_{o}=0$ & $m_{+}=m_{-}>0$ & $\langle\bar{\psi} \psi\rangle_{+}=\langle\bar{\psi} \psi\rangle_{-}>0$ & $B_{+}(0)>0, \tilde{B}_{-}(0)>0$ \\
$m_{0}>m_{e}>0$ & $m_{+}>-m_{-}>0$ & $\langle\bar{\psi} \psi\rangle_{+}>0>\langle\bar{\psi} \psi\rangle_{-}$ & $B_{+}(0)>0, B_{-}(0)<0$ \\
$m_{o}>0, m_{e}=0$ & $m_{+}=-m_{-}>0$ & $\langle\bar{\psi} \psi\rangle_{+}=-\langle\bar{\psi} \psi\rangle_{-}>0$ & $B_{+}(0)=-B_{-}(0)>0$ \\
$m_{o}=m_{e}>0$ & $m_{+}>0, m_{-}=0$ & $\langle\bar{\psi} \psi\rangle_{+}>0$, & $B_{+}(0)>0$, \\
& & $\langle\bar{\psi} \psi\rangle_{-}$undetermined & both $B_{-}(0)$ and $\tilde{B}_{-}(0)$
\end{tabular}

Therefore the two solutions $\left(B_{+}, B_{-}\right)$and $\left(B_{+}, \tilde{B}_{-}\right)$are realized in two different limits: the limit $m_{+}=-m_{-} \downarrow_{\widetilde{B}} 0$ leads to the solution $\left(B_{+}, B_{-}\right)$, whereas the limit $m_{+}=m_{-} \downarrow 0$ yields the solution $\left(B_{+}, \tilde{B}_{-}\right)$. This last set of solutions is of course only possible if the parameters $N, \theta$ are in the chirally broken phase.

From this consideration, the two sets of solutions cannot be realized simultaneously. The result which solution is realized depends on the ordering of taking the vanishing limit keeping the relation between $m_{e}$ and $m_{o}$. So in the presence of the CS term, taking the limit $m_{e}>m_{o} \downarrow 0$, we will get the solution $\left(B_{+}, \tilde{B}_{-}\right)$which has a higher value for the CJT effective potential than that of the "trivial" solution $\left(B_{+}, B_{-}\right)$. That means that this solution is a quasi-stable solution of the SD equation, and will eventually decay into the "trivial" solution. This quasi-stable solution does no longer exist beyond a certain value of $\theta$, the critical value $\theta_{c}(N)$ depending on $N$. At this point the system jumps directly to the state expressed by the solution $\left(B_{+}, B_{-}\right)$, which is energetically more favored, in a discontinuous way. 


\section{CONCLUSION AND DISCUSSION}

In (2+1)-dimensional QED with $N$ flavors of four-component Dirac fermions, we have solved the SD equation for the fermion propagator in the nonlocal gauge both analytically and numerically. In the absence of the bare CS term, we have shown the existence of a finite critical number of flavors $N_{c} \cong 4.3$, below which the chiral symmetry is spontaneously broken, in agreement with previous analyses [27,28, 16. In the presence of CS term, $\theta \neq 0$, we have obtained the critical line extending from the critical point $(N, \theta)=\left(N_{c}, 0\right)$ in the two-dimensional phase diagram $(N, \theta)$. Here a quite remarkable point is that, no matter how small $\theta$ is, this phase transition turns into a first-order transition when $\theta \neq 0$, although the critical point $\left(N_{c}, 0\right)$ in the absence of CS term is a continuous (infinite-order) phase transition point. Therefore the point $\left(N_{c}, 0\right)$ is the only one point on the critical line which exhibits continuous phase transition.

We have shown that the chiral-symmetric solution $\left(B_{+}, B_{-}\right)$and the chiral-symmetrybreaking one $\left(B_{+}, \tilde{B}_{-}\right)$of the SD equation are stationary points of the effective potential of the CJT type. Here it should be remarked that the trivial solution $\left(B_{+} \equiv 0, B_{-} \equiv 0\right)$ as a trivial stationary point (equivalently the trivial solution of the SD equation) is possible only when $\theta=0$. Although the symmetric solution $\left(B_{+}, B_{-}\right)$gives lower effective potential than the symmetry-breaking solution $\left(B_{+}, \tilde{B}_{-}\right)$, we have shown that it is possible to take the limit of removing the external source, $m_{e}, m_{o} \downarrow 0$, so that the spontaneously chiralsymmetry-breaking solution $\left(B_{+}, \tilde{B}_{-}\right)$is realized. However it is not yet clear whether they give local minima, local maxima, or possibly a saddle point of the effective potential and whether or not $\left(B_{+}, B_{-}\right)$gives the absolute minimum. In order to confirm this issue, it is necessary to calculate the second functional derivative:

$$
\frac{\delta^{2}}{\delta B_{ \pm}(p) \delta B_{ \pm}(q)} V_{C J T}\left[B_{+}, B_{-}\right]
$$

at the respective stationary point 42]. The system at an excited state $\left(B_{+}, \tilde{B}_{-}\right)$might be quasi-stable and might decay into the more stable state $\left(B_{+}, B_{-}\right)$in a finite time interval. The stability of the stationary point will be discussed in more detail in a subsequent paper.

It is interesting to see our result from the viewpoint of the lattice gauge theory where the lattice spacing $a$ corresponds to the inverse of the UV cutoff $\Lambda, a \sim 1 / \Lambda$. It is well known 44 that the nontrivial continuum limit can be possibly taken only at the secondand higher-order transition point where the correlation length diverges (in units of the lattice spacing). Hence our result implies that the meaningful continuum limit from the broken phase in the nonperturbatively regularized three-dimensional gauge field theory can be taken only at the point $\left(N_{c}, 0\right)$, i.e. in the absence of the bare CS term. This is a novel feature of the three-dimensional gauge field theory with a bare CS term, which seems to be overlooked so far. Our result may have important implications in the application of the three-dimensional gauge theory in condensed-matter physics as a long-wavelength effective theory of the microscopic model. This will be discussed in a forthcoming paper.

This result is obtained by using a consistent expansion of the full gauge boson propagator and the vertex in $1 / N$. In order to satisfy the WT identity (up to terms of order $1 / N$ and of order of the dynamically generated mass), we have adopted the nonlocal gauge function which allows us to neglect effect from the wave-function renormalization. A more accurate 
approximation would include a full $1 / N^{2}$ calculation, similar to the analysis in 28 in pure QED. Since in pure QED these $1 / N^{2}$ corrections do not change the result qualitatively, but only quantitatively (leading to a slightly different critical number of fermion flavors), we do not expect that those corrections will change our present result essentially. The question of gauge covariance can (in principle) be recovered by applying the Landau-Khalatnikov transformation rules to the various Green's functions 45. Therefore we expect our present results to hold also in a more elaborate approximation of the SD equation.

\section{ACKNOWLEDGEMENTS}

This work was initiated during a visit of one of the authors (P. M.) to Chiba University, and he would like to thank the members of the Graduate School of Science and Technology for their hospitality during that stay. We would like to thank K. Yamawaki, Yoonbai Kim, and T. Ebihara for stimulating discussions, and A. Shibata for helping with printing the postscript figures. P. M. has been financially supported by the JSPS (being a JSPS fellow under number 94146) and K.-I. K. is partly supported by the Computation Centre of Nagoya University.

\section{APPENDIX A: INTEGRATIONS}

\section{Angular Integration Formulae}

First we note the following relation

$$
\int_{0}^{\pi} \mathrm{d} \phi \sin \phi f(q)=\int_{-1}^{1} \mathrm{~d} z f(q)=\frac{1}{p k} \int_{|p-k|}^{p+k} q \mathrm{~d} q f(q)
$$

where $q^{2}=(p-k)^{2}=p^{2}+k^{2}-2 p k z$ with $z=\cos \phi$. Then we obtain the following formulae

$$
\begin{aligned}
\int_{-1}^{1} \mathrm{~d} z \frac{1}{q} & =\frac{2 \min (p, k)}{p k}, \\
\int_{-1}^{1} \mathrm{~d} z \frac{1}{q^{2}}= & \frac{1}{p k} \ln \frac{p+k}{|p-k|}, \\
\int_{-1}^{1} \mathrm{~d} z \frac{1}{q^{3}}= & \frac{1}{p k}\left(\frac{1}{|p-k|}-\frac{1}{p+k}\right), \\
\int_{-1}^{1} \mathrm{~d} z \frac{1}{q^{4}} \ln \left(1+\frac{q}{\alpha}\right)= & \frac{1}{p k}\left[\frac{1}{2 \alpha}\left(\frac{1}{|p-k|}-\frac{1}{p+k}\right)-\frac{1}{2 \alpha^{2}} \ln \frac{1+\frac{\alpha}{1+k \mid}}{1+\frac{\alpha}{p+k}}\right. \\
\int_{-1}^{1} \mathrm{~d} z \frac{1}{q(q+\alpha)}= & \frac{1}{p k} \ln \frac{p+k+\alpha}{|p-k|+\alpha} .
\end{aligned}
$$

For the calculation of the explicit CS term it is more convenient to use 


$$
\begin{aligned}
& \int_{-1}^{1} \mathrm{~d} z \frac{1}{q^{2}+p^{2}+2 p q z+M^{2}}=\frac{1}{2 p q} \ln \frac{(p+q)^{2}+M^{2}}{(p-q)^{2}+M^{2}}, \\
& \int_{-1}^{1} \mathrm{~d} z \frac{p q z}{q^{2}+p^{2}+2 p q z+M^{2}}=1+\frac{p^{2}+q^{2}+M^{2}}{4 p q} \ln \frac{(p+q)^{2}+M^{2}}{(p-q)^{2}+M^{2}} .
\end{aligned}
$$

In pure $\mathrm{QED}$ this gives for the angular integration

$$
\begin{aligned}
\mathrm{K}(p, k)= & \int_{-1}^{1} \mathrm{~d} z\left(\frac{2}{q(\alpha+q)}+\frac{2 \alpha}{q^{3}}-\frac{1}{q^{2}}-\frac{2 \alpha^{2} \ln \left(\frac{\alpha+q}{\alpha}\right)}{q^{4}}\right) \\
= & \frac{2 \alpha}{\max (p, k)\left|k^{2}-p^{2}\right|}+\frac{1}{k p} \ln \frac{\alpha+|k+p|}{\alpha+|k-p|} \\
& -\frac{\alpha^{2} \ln \left(1+\frac{|k-p|}{\alpha}\right)}{k p(-k+p)^{2}}+\frac{\alpha^{2} \ln \left(1+\frac{|k+p|}{\alpha}\right)}{k p(k+p)^{2}} .
\end{aligned}
$$

\section{Explicit Chern-Simons Term}

The explicit CS term, proportional to

$$
\begin{aligned}
I(p) & \equiv \int_{0}^{\infty} \frac{k^{2} \mathrm{~d} k}{k^{2}+M_{ \pm}^{2}} \int_{-1}^{1} \mathrm{~d} z \frac{k \cdot(k-p)}{(k-p)^{2}(|k-p|+\alpha)^{2}} \\
& =\int_{0}^{\infty} \frac{\mathrm{d} q}{(q+\alpha)^{2}} \int_{-1}^{1} \mathrm{~d} z \frac{(q+p) \cdot q}{(q+p)^{2}+M_{ \pm}^{2}},
\end{aligned}
$$

can be calculated analytically. The angular integration gives

$$
I(p)=\int_{0}^{\infty} \frac{\mathrm{d} q}{(q+\alpha)^{2}}\left(1-\frac{p^{2}-q^{2}+M_{ \pm}^{2}}{4 p q} \ln \frac{(p+q)^{2}+M_{ \pm}^{2}}{(p-q)^{2}+M_{ \pm}^{2}}\right),
$$

which we can calculate approximately by expanding the logarithm for $p<q$ and $p>q$. Taking into account only the leading-order terms in $\min (p, q) / \max (p, q)$ gives

$$
I(p)=\frac{3 M_{ \pm}^{2}+p^{2}}{3\left(M_{ \pm}^{2}+p^{2}\right)^{2}} \int_{0}^{p} \frac{2 q^{2} \mathrm{~d} q}{(q+\alpha)^{2}}+\int_{p}^{\infty} \frac{\mathrm{d} q}{(q+\alpha)^{2}} \frac{2 q^{2}}{M_{ \pm}^{2}+q^{2}},
$$

which can easily be calculated. Expanding the result for $M_{ \pm} \ll \alpha$ gives

$$
\begin{aligned}
I(p)= & 2 \frac{3 M_{ \pm}^{2}+p^{2}}{3\left(M_{ \pm}^{2}+p^{2}\right)^{2}}\left(\frac{p^{2}+2 \alpha p}{\alpha+p}-2 \alpha \ln (1+p / \alpha)\right) \\
& +2\left(\frac{1}{(\alpha+p)}-\frac{M_{ \pm}}{\alpha^{2}}\left(\frac{\pi}{2}-\arctan \left(\frac{p}{M_{ \pm}}\right)\right)+\frac{M_{ \pm}^{2}}{\alpha^{3}} \ln \frac{(\alpha+p)^{2}}{\left(M_{ \pm}^{2}+p^{2}\right)}\right) .
\end{aligned}
$$

However, we are only interested in the leading-order behavior in the infrared and ultraviolet region, so we finally get for $p \ll \alpha$

$$
I(p)=2+\mathcal{O}(p / \alpha)+\mathcal{O}\left(M_{ \pm} / \alpha\right),
$$

or $p \gg \alpha \gg M_{ \pm}$

$$
I(p)=\frac{8 \alpha}{3 p}+\mathcal{O}\left(\alpha^{2} / p^{2}\right)+\mathcal{O}\left(M_{ \pm} / p\right)
$$




\section{APPENDIX B: UV EXPANSION OF THE HYPERGEOMETRIC FUNCTION}

\section{Pure QED}

In pure QED, the ultraviolet boundary condition, Eq. 53, leads to the requirement

$$
{ }_{2} F_{1}\left(a_{+}, a_{-}, \frac{3}{2} ; \frac{-\alpha^{2}}{m^{2}}\right)+\alpha{ }_{2} F_{1}^{\prime}\left(a_{+}, a_{-}, \frac{3}{2} ; \frac{-\alpha^{2}}{m^{2}}\right)=\frac{8}{3 \pi^{2} N}{ }_{2} F_{1}\left(a_{+}, a_{-}, \frac{3}{2} ; \frac{-\alpha^{2}}{m^{2}}\right),
$$

with $a_{ \pm}=\frac{1}{4} \pm \frac{1}{4} i \sqrt{N_{c} / N-1}$ and $N_{c}=128 /\left(3 \pi^{2}\right)$. The derivative w.r.t. $\alpha$ of the hypergeometric function gives

$$
{ }_{2} F_{1}\left(a_{+}, a_{-}, \frac{3}{2} ; \frac{-\alpha^{2}}{m^{2}}\right)+\alpha{ }_{2} F_{1}^{\prime}\left(a_{+}, a_{-}, \frac{3}{2} ; \frac{-\alpha^{2}}{m^{2}}\right)={ }_{2} F_{1}\left(a_{+}, a_{-}, \frac{1}{2} ; \frac{-\alpha^{2}}{m^{2}}\right),
$$

so we arrive at

$$
{ }_{2} F_{1}\left(a_{+}, a_{-}, \frac{1}{2} ; \frac{-\alpha^{2}}{m^{2}}\right)=a_{+} a_{-2} F_{1}\left(a_{+}, a_{-}, \frac{3}{2} ; \frac{-\alpha^{2}}{m^{2}}\right),
$$

where we have used $8 /\left(3 \pi^{2} N\right)=a_{+} a_{-}$. To derive the behavior of the mass $m$ close to the critical value, we expand Eq. (B1) in $\alpha / m$. Taking into account leading order only, we find

$$
\begin{aligned}
& { }_{2} F_{1}\left(a_{+}, a_{-}, \frac{1}{2} ; \frac{-\alpha^{2}}{m^{2}}\right)=\frac{\Gamma(1 / 2) \Gamma\left(a_{-}-a_{+}\right)}{\Gamma\left(a_{-}\right) \Gamma\left(1 / 2-a_{+}\right)}\left(\frac{\alpha}{m}\right)^{-2 a_{+}}+\text {h.c. } \\
& { }_{2} F_{1}\left(a_{+}, a_{-}, \frac{3}{2} ; \frac{-\alpha^{2}}{m^{2}}\right)=\frac{\Gamma(3 / 2) \Gamma\left(a_{-}-a_{+}\right)}{\Gamma\left(a_{-}\right) \Gamma\left(3 / 2-a_{+}\right)}\left(\frac{\alpha}{m}\right)^{-2 a_{+}}+\text {h.c. }
\end{aligned}
$$

We are interested in $m / \alpha$, so we rewrite the boundary condition into

$$
\left(\frac{m}{\alpha}\right)^{i \omega}=\frac{\Gamma\left(1+\frac{i}{2} \omega\right) \Gamma\left(a_{-}\right)^{2}\left(1-\frac{1}{2} a_{-}\right)}{\Gamma\left(1-\frac{i}{2} \omega\right) \Gamma\left(a_{+}\right)^{2}\left(1-\frac{1}{2} a_{+}\right)}
$$

using the notation $\omega=\sqrt{N_{c} / N-1}$. Since the absolute value of the RHS is equal to one, we can write it as $e^{2 i(\phi-n \pi)}$, with

$$
\phi=\arg \left[\Gamma\left(1+\frac{i}{2} \omega\right) \Gamma\left(a_{-}\right)^{2}\left(1-\frac{1}{2} a_{-}\right)\right],
$$

and $n$ integer. This leads to the equation for $m / \alpha$

$$
\frac{m}{\alpha}=\exp \frac{-2 n \pi+2 \phi}{\omega} .
$$

Next, we expand $\phi$ leading order in $\omega$, using a Taylor expansion and the Euler constant $\gamma$

$$
\begin{aligned}
\Gamma\left(1 \pm \frac{i}{2} \omega\right) & =1 \mp \frac{i}{2} \omega \gamma+\mathcal{O}\left(\omega^{2}\right) \\
\Gamma\left(a_{ \pm}\right) & =\Gamma(1 / 4)\left(1 \mp\left(\gamma+\frac{1}{2} \pi+3 \ln 2\right) \frac{i}{4} \omega\right)+\mathcal{O}\left(\omega^{2}\right),
\end{aligned}
$$

which leads to 


$$
\phi \simeq\left(\frac{1}{4} \pi+\frac{3}{2} \ln 2+\frac{1}{7}\right) \omega
$$

In this way we arrive finally at

$$
\frac{m}{\alpha} \simeq \exp \left[\frac{-2 n \pi}{\omega}+\frac{1}{2} \pi+3 \ln 2+\frac{2}{7}\right] .
$$

This expression is only valid for $m \ll \alpha$, which means that $n$ has to be positive. Using the effective potential, one can show that the largest value for $m / \alpha$ gives the lowest energy, therefore the ground state corresponds to $n=1$, and higher excited (oscillating) solutions are given by $n=2,3,4, \ldots$.

For the chiral condensate we need to know the mass function at momentum $p=\alpha, m(\alpha)$. Using the same expansions as above, we have

$$
m(\alpha)=m\left(\frac{2 i \Gamma(3 / 2) \Gamma\left(1-\frac{i}{2} \omega\right)}{\omega \Gamma\left(a_{-}\right) \Gamma\left(3 / 2-a_{+}\right)}\left(\frac{\alpha}{m}\right)^{-2 a_{+}}+\text {h.c. }\right),
$$

with $m$ given by Eq. (B8). Close to the critical coupling we can expand this in $\omega$, using

$$
\left(\frac{m}{\alpha}\right)^{\frac{i}{2} \omega}=e^{i(-\pi+\phi)}=-(\cos \phi+i \sin \phi),
$$

which leads to

$$
\frac{m(\alpha)}{\alpha} \simeq-8 \frac{\Gamma(1 / 2)}{\Gamma(1 / 4)^{2}}\left(\frac{m}{\alpha}\right)^{3 / 2}\left(\left(-1+\frac{\pi}{4}+\frac{3}{2} \ln 2\right) \cos \phi-\frac{\sin \phi}{\omega}\right) .
$$

Finally, using the expansion for $\phi$, we find

$$
\frac{m(\alpha)}{\alpha} \simeq \frac{64}{7} \frac{\Gamma(1 / 2)}{\Gamma(1 / 4)^{2}}\left(\frac{m}{\alpha}\right)^{3 / 2}
$$

with $m / \alpha$ given by Eq. (B12).

\section{Chern-Simons Term: critical mass as function of $N$}

The equation for $M_{c}$ as function of $N$, Eq. 89, is

$$
\begin{aligned}
{ }_{2} F_{1}\left(a_{+}, a_{-}, \frac{-1}{2} ;-\alpha^{2} / M_{c}^{2}\right) & +a_{+} a_{-2} F_{1}\left(a_{+}, a_{-}, \frac{1}{2} ;-\alpha^{2} / M_{c}^{2}\right) \\
& -2 a_{+} a_{-2} F_{1}\left(a_{+}, a_{-}, \frac{3}{2} ;-\alpha^{2} / M_{c}^{2}\right)=0 .
\end{aligned}
$$

We can expand this as usual for small $M_{c}$

$$
\begin{gathered}
\left(\frac{\alpha}{M_{c}}\right)^{-2 a_{+}}\left(\frac{\Gamma(-1 / 2) \Gamma\left(a_{-}-a_{+}\right)}{\Gamma\left(a_{-}\right) \Gamma\left(-1 / 2-a_{+}\right)}+a_{+} a_{-} \frac{\Gamma(1 / 2) \Gamma\left(a_{-}-a_{+}\right)}{\Gamma\left(a_{-}\right) \Gamma\left(1 / 2-a_{+}\right)}\right. \\
\left.-2 a_{+} a_{-} \frac{\Gamma(3 / 2) \Gamma\left(a_{-}-a_{+}\right)}{\Gamma\left(a_{-}\right) \Gamma\left(3 / 2-a_{+}\right)}\right)+ \text {h.c. }=0,
\end{gathered}
$$


which can be reduced to

$$
\left(\frac{\alpha}{M_{c}}\right)^{-2 a_{+}} \frac{\Gamma\left(a_{-}-a_{+}\right)}{\Gamma\left(a_{-}\right)^{2}}\left(1+a_{+}+a_{+} a_{-}\right)+\text {h.c. }=0,
$$

so we arrive at

$$
\left(\frac{M_{c}}{\alpha}\right)^{i \omega}=\frac{\Gamma\left(1+\frac{i}{2} \omega\right) \Gamma\left(a_{-}\right)^{2}\left(1+a_{-}+a_{+} a_{-}\right)}{\Gamma\left(1-\frac{i}{2} \omega\right) \Gamma\left(a_{+}\right)^{2}\left(1+a_{+}+a_{+} a_{-}\right)} .
$$

Again, we can write this as

$$
\frac{M_{c}}{\alpha}=\exp \frac{-2 n \pi+2 \phi}{\omega}
$$

but now with

$$
\phi=\arg \left[\Gamma\left(1+\frac{i}{2} \omega\right) \Gamma\left(a_{-}\right)^{2}\left(1+a_{-}+a_{+} a_{-}\right)\right] .
$$

Finally we expand $\phi$ in $\omega$

$$
\phi \simeq\left(\frac{1}{4} \pi+\frac{3}{2} \ln 2-\frac{4}{21}\right) \omega
$$

so we arrive at

$$
\frac{M_{c}}{\alpha}=\exp \left[\frac{-2 n \pi}{\omega}+\frac{1}{2} \pi+3 \ln 2-\frac{8}{21}\right],
$$

with $n=1,2,3, .$. ; the term $-2 n \pi / \omega$ arises just as in pure QED, and also here there are infinitely many solutions for each value of $N$. The interesting one is the one corresponding to the ground state in pure QED, $n=1$.

\section{Chern-Simons Term: critical $\theta$ as function of $N$}

Using the above approximation for $M_{c}$ as function of $N$, we can now calculate $\theta_{c}$ as function of $N$, see Eq. 86. In general we have

$$
\theta_{c}=\frac{ \pm \pi^{2} N}{8\left(1+\frac{16}{9 \pi^{2} N}\right)}\left(M_{c 2} F_{1}\left(a_{+}, a_{-}, \frac{1}{2} ;-\alpha^{2} / M_{c}^{2}\right)-a_{+} a_{-} M_{c 2} F_{1}\left(a_{+}, a_{-}, \frac{3}{2} ;-\alpha^{2} / M_{c}^{2}\right)\right),
$$

which we can expand

$$
\frac{\theta_{c}}{\alpha}= \pm \frac{\pi^{2} N \Gamma(1 / 2)}{8\left(1+\frac{16}{9 \pi^{2} N}\right)}\left(\frac{M_{c}}{\alpha}\right)^{3 / 2}\left(\left(\frac{M_{c}}{\alpha}\right)^{\frac{i}{2} \omega} \frac{\Gamma\left(a_{-}-a_{+}\right)}{\Gamma\left(a_{-}\right)^{2}}\left(1-\frac{1}{2} a_{+}\right)+\text {h.c. }\right) .
$$

We expand the $\Gamma$ functions as before in $\omega$ and use

$$
\left(\frac{M_{c}}{\alpha}\right)^{\frac{i}{2} \omega}=e^{i(-\pi+\phi)}=-(\cos \phi+i \sin \phi),
$$

which we have just derived, to get

$$
\frac{\theta_{c}}{\alpha} \simeq \mp \frac{448}{75} \frac{\Gamma(1 / 2)}{\Gamma(1 / 4)^{2}}\left(\frac{M_{c}}{\alpha}\right)^{3 / 2}
$$

to leading order in $\omega$, using the expression for $\phi$, Eq. B23. 


\section{APPENDIX C: STATIONARY POINT OF THE EFFECTIVE POTENTIAL}

The effective potential given in section $\nabla]$ reproduces the SD equation for $B_{ \pm}$at the stationary point as follows. The variation with respect to $B_{ \pm}$of the effective potential is calculated as follows:

$$
\begin{aligned}
\frac{\delta}{\delta B_{ \pm}(p)} V_{0}[B] & =\frac{\delta}{\delta B_{ \pm}(p)} \int \frac{\mathrm{d}^{3} p}{(2 \pi)^{3}}\left[-\ln \left(1+\frac{B_{ \pm}^{2}(p)}{p^{2}}\right)+2 \frac{B_{ \pm}^{2}(p)}{p^{2}+B_{ \pm}^{2}(p)}\right] \\
& =\frac{1}{(2 \pi)^{3}} 2 B_{ \pm}(p) \frac{\left[p^{2}-B_{ \pm}^{2}(p)\right]}{\left[p^{2}+B_{ \pm}^{2}(p)\right]^{2}}
\end{aligned}
$$

and

$$
\begin{aligned}
\frac{\delta}{\delta B_{ \pm}(p)} V_{1}[B]= & -\frac{2 e^{2}}{(2 \pi)^{3}} \frac{\left[p^{2}-B_{ \pm}^{2}(p)\right]}{\left[p^{2}+B_{ \pm}^{2}(p)\right]^{2}} \int \frac{\mathrm{d}^{3} k}{(2 \pi)^{3}} \frac{1}{k^{2}+B_{ \pm}^{2}(k)} \\
& \times\left[\left(2 D_{T}(q)+\frac{a(q)}{q^{2}}\right) B_{ \pm}(k) \mp 2 \frac{(k \cdot q)}{|q|} D_{O}(q)\right] .
\end{aligned}
$$

Therefore we obtain

$$
\begin{aligned}
\frac{\delta}{\delta B_{ \pm}(p)} V[B]= & \frac{2}{(2 \pi)^{3}} \frac{\left[p^{2}-B_{ \pm}^{2}(p)\right]}{\left[p^{2}+B_{ \pm}^{2}(p)\right]^{2}}\left\{B_{ \pm}(p)-e^{2} \int \frac{\mathrm{d}^{3} k}{(2 \pi)^{3}} \frac{1}{k^{2}+B_{ \pm}^{2}(k)}\right. \\
& \left.\times\left[\left(2 D_{T}(q)+\frac{a(q)}{q^{2}}\right) B_{ \pm}(k) \mp 2 \frac{(k \cdot q)}{|q|} D_{O}(q)\right]\right\} .
\end{aligned}
$$

This shows that the SD equation for $B_{ \pm}$is obtained from the stationary condition $\delta V[B] / \delta B_{ \pm}=0$ of the CJT effective potential $V[B]$. 


\section{REFERENCES}

* e-mail: kondo@cuphd.nd.chiba-u.ac.jp

$\dagger \quad$ e-mail: maris@eken.phys.nagoya-u.ac.jp

[1] K.-I. Kondo and P. Maris, First-order Phase Transition in Three-dimensional QED with Chern-Simons Term, Chiba/Nagoya Univ. Preprint, CHIBA-EP-84/DPNU-94-33, (hep-ph/9408210), to be published in Phys. Rev. Lett.

[2] T. Appelquist, M. Bowick, D. Karabali and L.C.R. Wijewardhana, Phys. Rev. D 33 (1986) 3704.

[3] R.D. Pisarski, Phys. Rev. D29 (1984) 2423.

[4] G.W. Semenoff and L.C.R. Wijewardhana, Phys. Rev. Lett. 62 (1988) 2633.

[5] V.A. Miransky, Nuovo Cimento 90A (1985) 149.

[6] S. Deser, R. Jackiw and S. Templeton, Ann. Phys. 140 (1981) 372.

[7] E. Fradkin, Field Theories of Condensed Matter Systems, (Addison-Wesley Publishing Company, 1991).

[8] F. Wilczek, Fractional Statistics and Anyon Superconductivity, (World Scientific, 1990)

[9] N. Dorey and N.E. Mavromatos, Nucl. Phys. B 386 (1992) 614.

[10] T. Appelquist, M.J. Bowick, D. Karabali and L.C.R. Wijewrdhana, Phys. Rev. D 33 (1986) 3774.

[11] A.P. Polychronakos, Phys. Rev. Lett. 60 (1988) 1920.

[12] S. Rao and R. Yahalom, Phys. Lett. B 172 (1986) 227; Phys. Rev. D 34 (1986) 1194.

[13] Y. Hoshino and T. Matsuyama, Phys. Lett. B 222 (1989) 493.

[14] C. Vafa and E. Witten, Nucl. Phys. B 234 (1984) 173.

[15] M. Carena, T.E. Clark and C.E.M. Wagner, Nucl. Phys. B 356 (1991) 117; Phys. Lett. B 259 (1991) 128.

[16] K.-I. Kondo, T. Ebihara, T. Iizuka and E. Tanaka, Dynamical Breakdown of Chirality and Parity in (2+1)-dimensional QED, Chiba Univ. Preprint, CHIBA-EP-77-REV, (hep-ph/9404361, revised), to be published in Nucl. Phys. B.

[17] For a review, see C.D. Roberts and A.G. Williams, Progr. Part. and Nucl. Phys. 33 (1994) 477, and the Proceedings of the 1991 Nagoya Spring School on Dynamical Symmetry Breaking, K. Yamawaki (editor), (World Scientific, Singapore, 1992).

[18] K.-I. Kondo and H. Nakatani, Mod. Phys. Lett. A4 (1989) 2155.

[19] K.-I. Kondo and H. Nakatani, Mod. Phys. Lett. A 5 (1990) 407.

[20] E. Dagotto, J.B. Kogut and A. Kocić, Phys. Rev. Lett. 62 (1989) 1083-1086; E. Dagotto, A. Kocić and J.B. Kogut, Nucl. Phys. B 334 (1990) 279.

[21] D.C. Curtis, M.R. Pennington and D. Walsh, Phys. Lett. B 295 (1992) 313; M.R. Pennington and D. Walsh, Phys. Lett. B 253 (1991) 246.

[22] D. Atkinson, P.W. Johnson and P. Maris, Phys. Rev. D 42 (1990) 602.

[23] D.C. Curtis and M.R. Pennington, Phys. Rev. D 44 (1991) 536.

[24] D. Atkinson, V.P. Gusynin and P. Maris, Phys. Lett. B 303 (1993) 157; D. Atkinson, J.C.R. Bloch, V.P. Gusynin, M.R. Pennington and M. Reenders, Phys. Lett. B 329 (1994) 117;

[25] K.-I. Kondo, Intern. J. Mod. Phys. A 7 (1992) 7239.

[26] R. Jackiw and S. Templeton, Phys. Rev. D23 (1981) 2291; T.W. Appelquist and R.D. Pisarski, Phys. Rev. D23 (1981) 2305; T.W. Appelquist and U. Heinz, Phys. Rev. D23 (1981) 2169. 
[27] T. Appelquist, D. Nash and L.C.R. Wijewardhana, Phys. Rev. Lett. 60 (1988) 2575.

[28] D. Nash, Phys. Rev. Lett. 62 (1989) 3024.

[29] M.R. Pennington and S.P. Webb, Hierarchy of scales in three dimensional QED, BNL40886, January 1988 (unpublished).

[30] D. Atkinson, P.W. Johnson and M.R. Pennington, Dynamical mass generation in threedimensional QED, BNL-41615, August 1988 (unpublished).

[31] V. Azcoiti, X.-Q. Luo, C.E. Piedratita, G. Di Carlo, A.F. Grillo and A. Galante, Phys. Lett. B 313 (1993) 180.

[32] H. Nakatani, Comment given at the 1988 International Workshop on New Trend in Strong Coupling Gauge Theories, Nagoya, Aug. 24-27 (unpublished).

[33] H. Georgi, E.H. Simmons and A.G. Cohen, Phys. Lett. B 236 (1990) 183; E.H. Simmons, Comment on higher-order corrections in (2+1)-dimensional QED, NSF-ITP-9026, HUTP-90/A009.

[34] T. Kugo and M.G. Mitchard, Phys. Lett. B 282 (1992) 162.

[35] K.-I. Kondo and H. Nakatani, Prog. Theor. Phys. 87 (1992) 193.

[36] J. Cornwall, R. Jackiw and E. Tomboulis, Phys. Rev. D 10 (1974) 2428.

[37] D. Atkinson, J. Math. Phys. 28 (1987) 2494; D. Atkinson, P.W. Johnson and K. Stam, Phys. Lett. B 201 (1988) 105.

[38] K.-I. Kondo, Triviality Problem and Schwinger-Dyson Equation Approach, in Dynamical Symmetry Breaking, ed. K. Yamawaki (World Scientific Pub. Co., Singapore, 1992).

[39] P. Maris, Nonperturbative analysis of the fermion propagator: complex singularities and dynamical mass generation, Ph.D. Thesis, U. of Groningen, October 1993.

[40] D.K. Hong and S.H. Park, Phys. Rev. D47 (1993) 3651.

[41] R.W. Haymaker, Rivista del Nuovo Cimento, 14 (1991) No. 8, 1.

[42] T. Matsuki, Z. Phys. C51 (1991) 429; Stability at the origin in (2+1)-dimensional QED, in 1989 Workshop on Dynamical Symmetry Breaking, Nagoya University, 1989.

[43] T. Matsuki, L. Miao and K.S. Viswanathan, Simon Fraser Univ. Preprint, June 1987 (revised: May 1988).

[44] See for example, M. Creutz, Quarks, Gluons and Lattices, (Cambridge University Press, 1983); E. Seiler, Gauge Theories as a Problem of Constructive Quantum Field Theory and Statistical Mechanics, Lecture Notes in Physics 159 (Springer Verlag, 1982).

[45] L.D. Landau and I.M. Khalatnikov, J. Exper. Theor. Phys. USSR 29, 89, (1955), (translation: Sov. Phys. JETP 2, 69 (1956)). 


\section{FIGURES}

FIG. 1. Scaling of the fermion mass and the chiral condensates: the quantities $\omega \ln (m(0) / \alpha)$ and $\omega \ln \left(\langle\bar{\psi} \psi\rangle / \alpha^{2}\right)$ as functions of $\omega=\sqrt{N_{c} / N-1}$ for the linearized equation (analytical solution, dashed line) and the full nonlinear integral equation (numerically, solid line) in pure QED.

FIG. 2. The fermion mass function $m(p)$ in pure QED: the analytical solution (dashed line) and numerical one (solid line) for $N=2$ and $N=3$ where both the horizontal and the vertical axes are logarithmic.

FIG. 3. The numerical solutions for wave-function renormalization functions, $A_{+}(p)$ and $\tilde{A}_{-}(p)$, and mass functions, $B_{+}(p), \tilde{B}_{-}(p), B_{e}(p), B_{o}(p)$ at $\theta=0.4 \cdot 10^{-5}$ and $m(p)$ at $\theta=0$ for $N=3$. (a) $1-A_{+}(p), 1-\tilde{A}_{-}(p)$, (b) mass functions in the IR region, (c) the same as (b) in the intermediate region.

FIG. 4. The infrared values $B_{+}(0)$ (dashed line) and $\tilde{B}_{-}(0)$ (solid line) as functions of $N$ for some different values of $\theta$, obtained numerically by solving the two sets of coupled integral equations. The dotted line indicates $m(0)$ in the case of $\theta=0$.

FIG. 5. The infrared values for $B_{+}(0), \tilde{B}_{-}(0), B_{e}(0), B_{o}(0)$, and the chiral condensate $\langle\bar{\psi} \psi\rangle$ as functions of $\theta$ for $N=3$, obtained numerically by solving the two sets of coupled integral equations. Note that the scale for the condensate is different.

FIG. 6. The infrared values $B_{+}(0)$ (dashed lines) and $\tilde{B}_{-}(0)$ (solid lines) as obtained analytically: (a) as function of $\theta$ for $N=3$, rescaled and compared with our numerical solutions, and (b) as function of $N$ for some different values of $\theta$, which should be compared with the numerical results plotted in Fig. 4 .

FIG. 7. Phase diagram in the $(N, \theta)$-plane: the critical line for the chiral phase transition obtained analytically and some numerical estimates for the critical point.

FIG. 8. The chiral condensate and $B_{e}(0)$ as functions of $N$ for $\theta=10^{-5}$ and for pure QED $(\theta=0)$, obtained numerically and analytically. Note that the scale for the condensate and $B_{e}(0)$ is different.

FIG. 9. The analytical solution of the linearized equation and the numerical solution of the full integral equation for $N=3$ and $\theta=0.4 \cdot 10^{-5}$. 
This figure "fig1-1.png" is available in "png" format from: http://arxiv.org/ps/hep-ph/9501280v1 
Figure 1

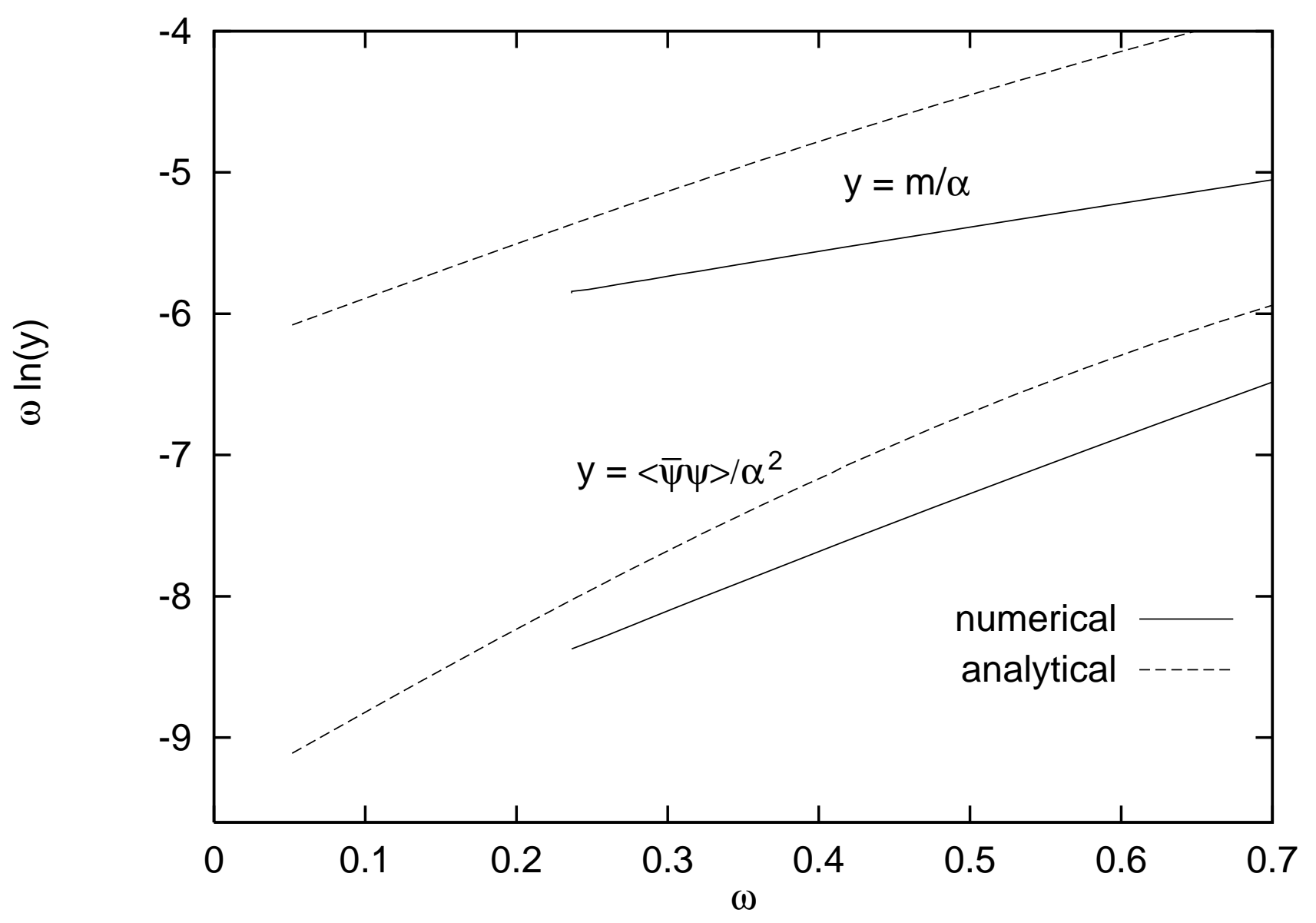


This figure "fig2-1.png" is available in "png" format from: http://arxiv.org/ps/hep-ph/9501280v1 
This figure "fig3-1.png" is available in "png" format from: http://arxiv.org/ps/hep-ph/9501280v1 
This figure "fig4-1.png" is available in "png" format from: http://arxiv.org/ps/hep-ph/9501280v1 
This figure "fig5-1.png" is available in "png" format from: http://arxiv.org/ps/hep-ph/9501280v1 
This figure "fig1-2.png" is available in "png" format from: http://arxiv.org/ps/hep-ph/9501280v1 
This figure "fig2-2.png" is available in "png" format from: http://arxiv.org/ps/hep-ph/9501280v1 
Figure 2

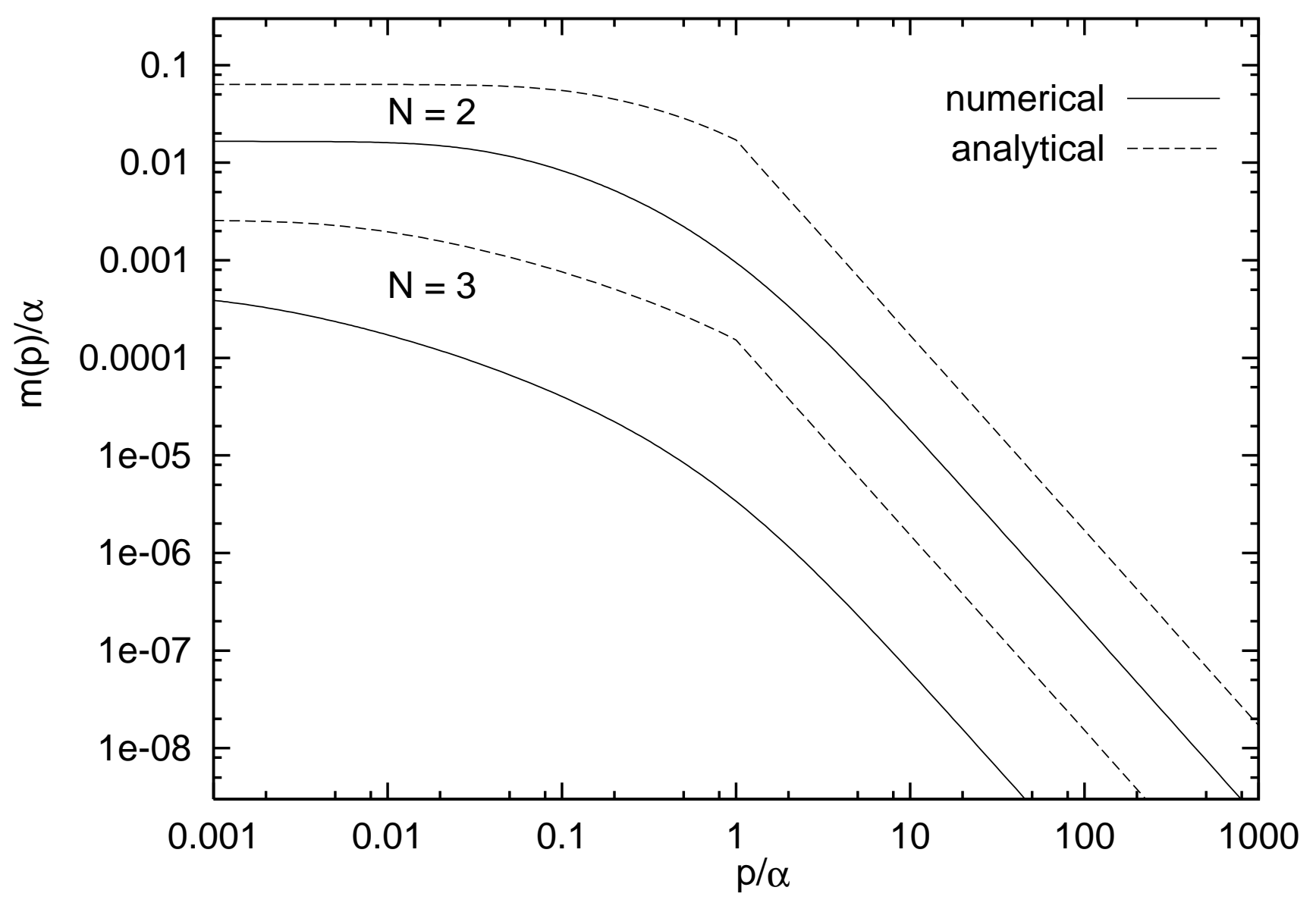


This figure "fig3-2.png" is available in "png" format from: http://arxiv.org/ps/hep-ph/9501280v1 
This figure "fig4-2.png" is available in "png" format from: http://arxiv.org/ps/hep-ph/9501280v1 
This figure "fig5-2.png" is available in "png" format from: http://arxiv.org/ps/hep-ph/9501280v1 
Figure $3 a$

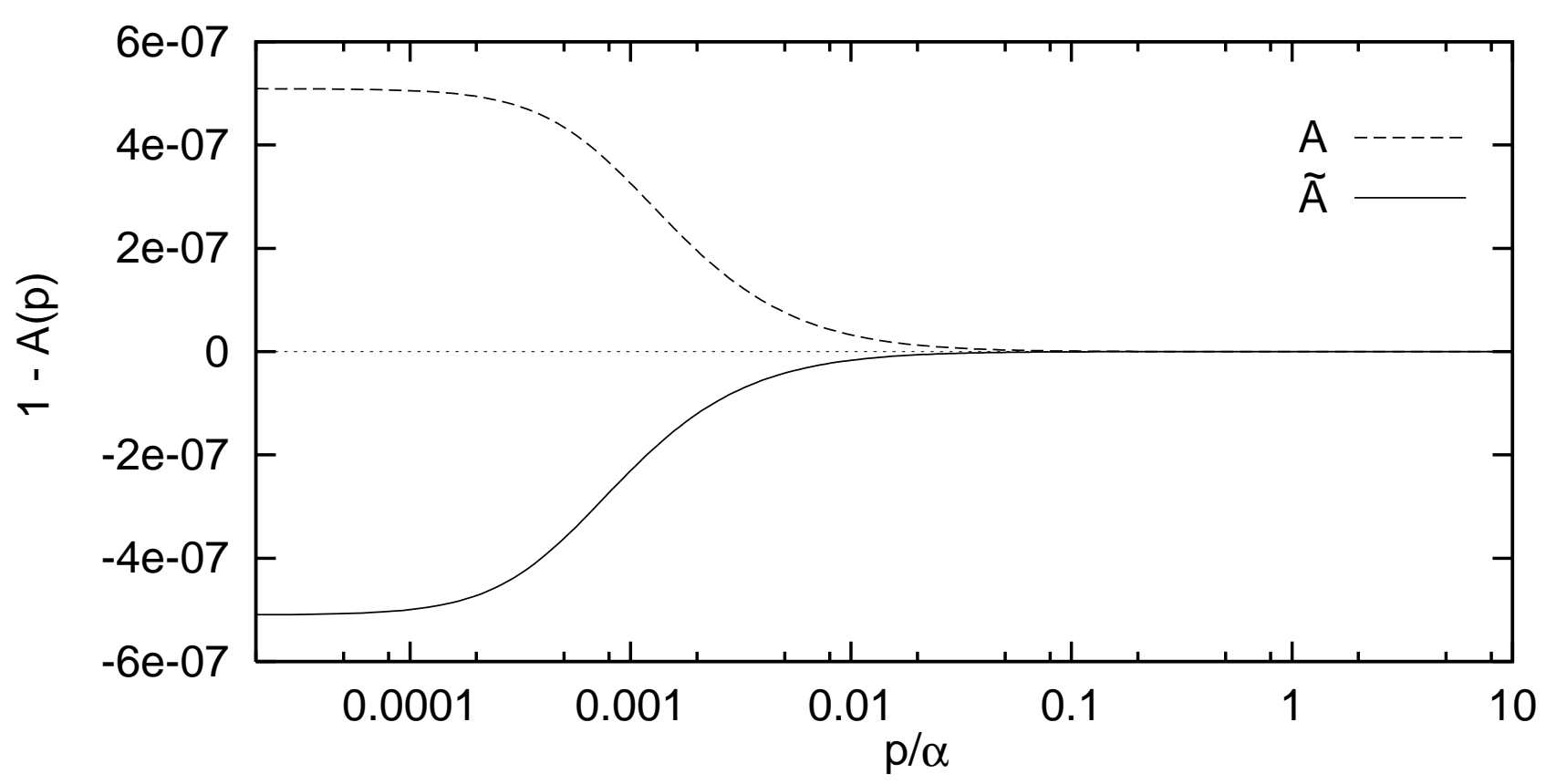

Figure 3b

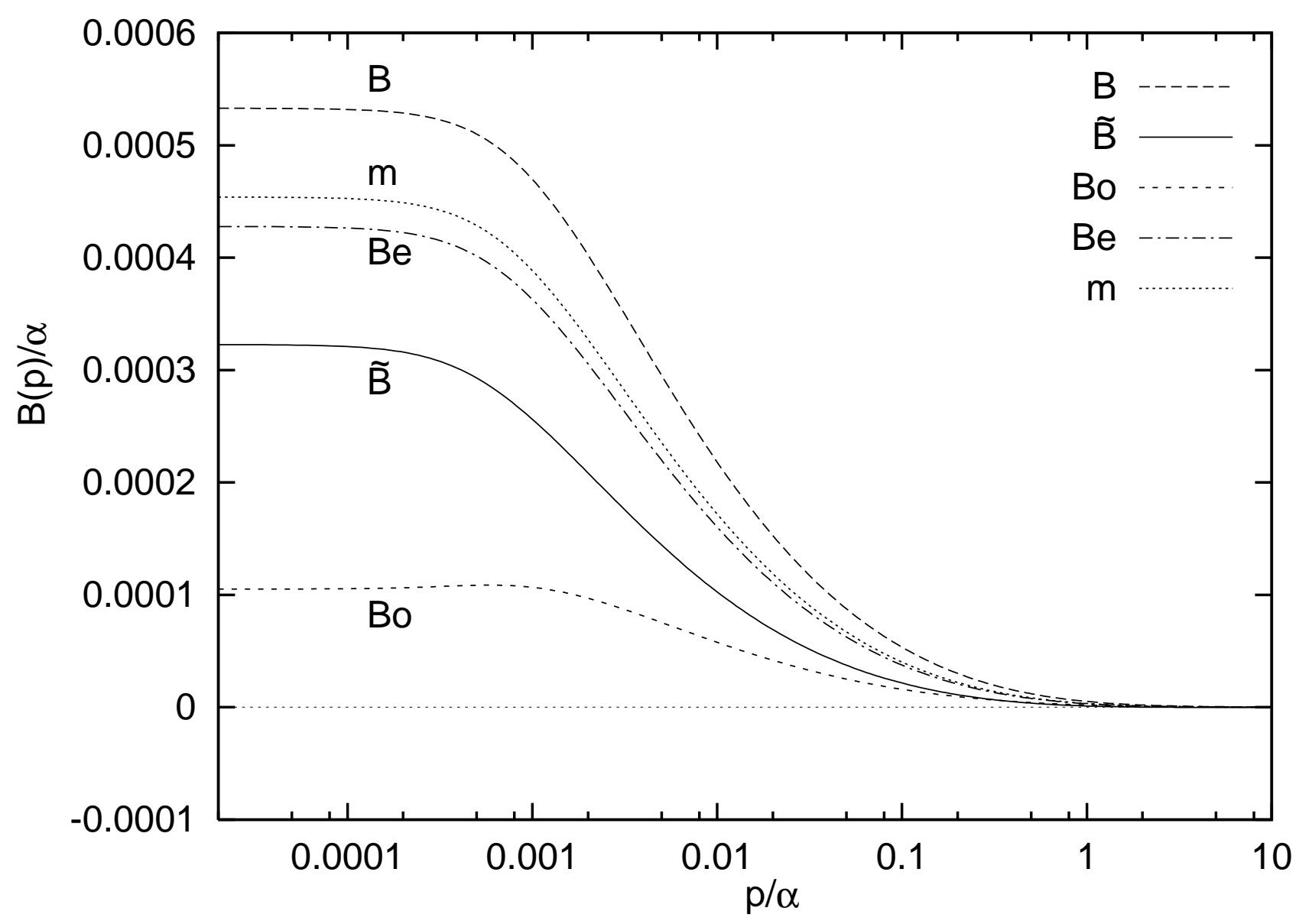


Figure 3c

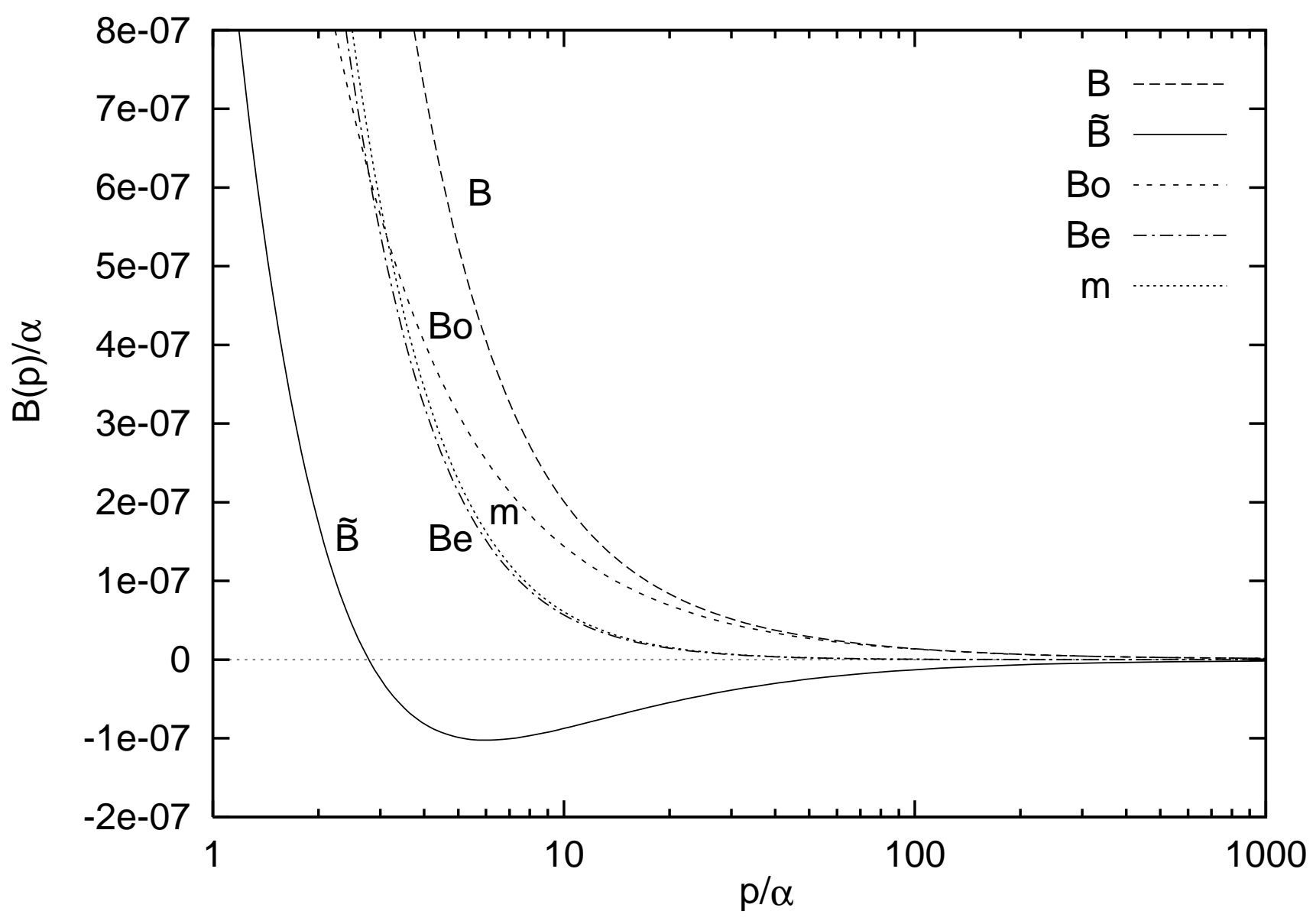


This figure "fig5-3.png" is available in "png" format from: http://arxiv.org/ps/hep-ph/9501280v1 
Figure 4

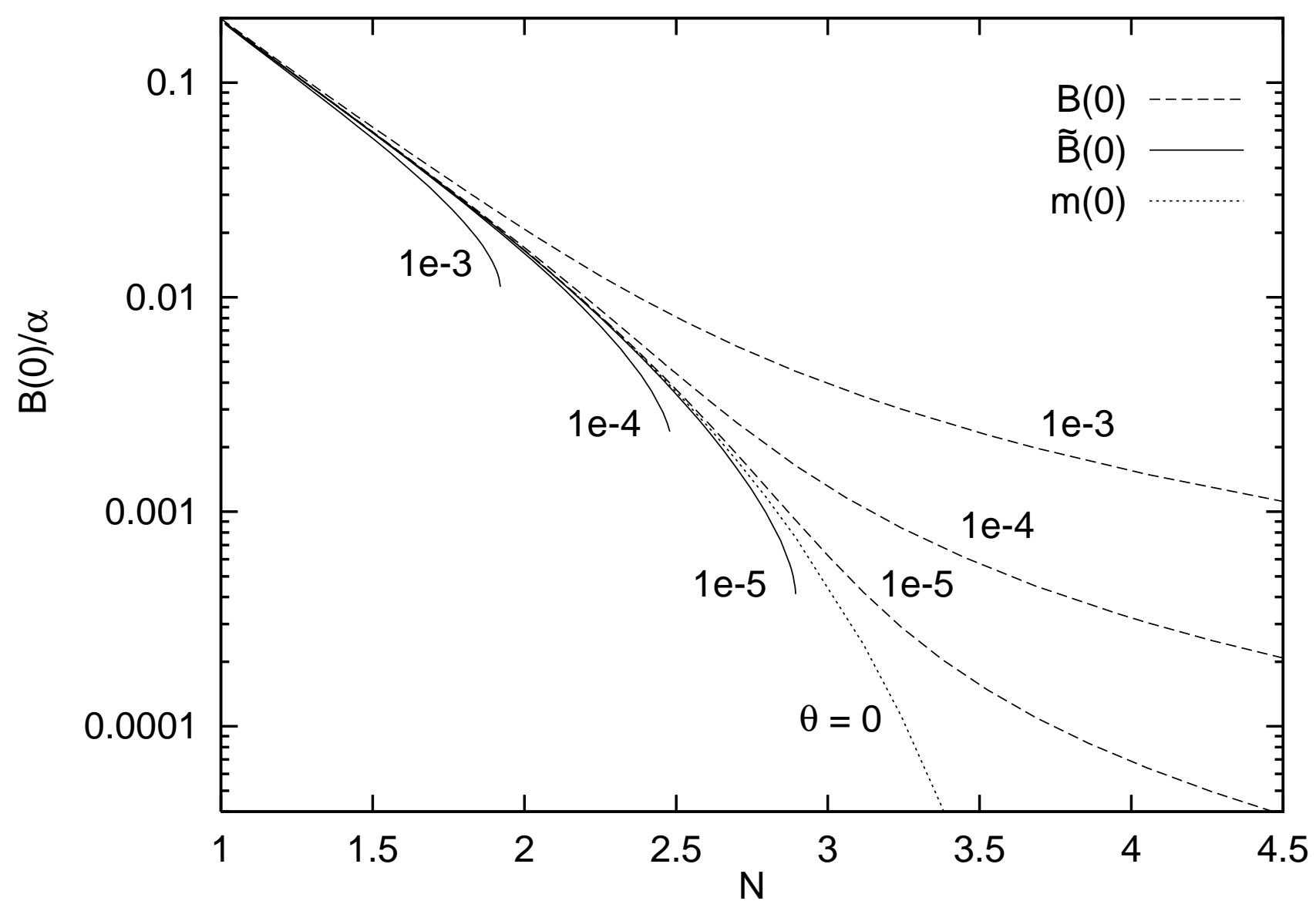


Figure 5

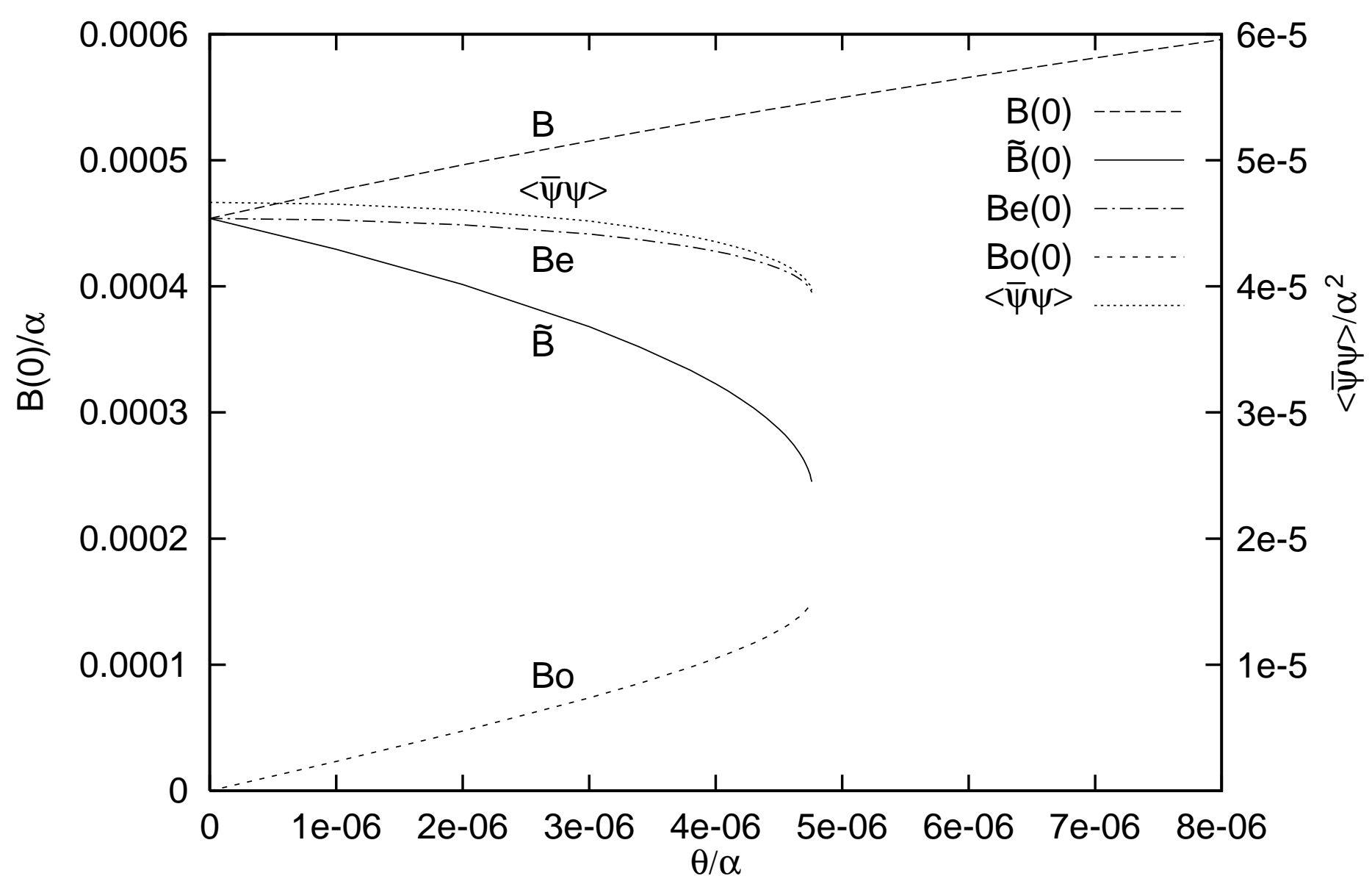


Figure $6 a$

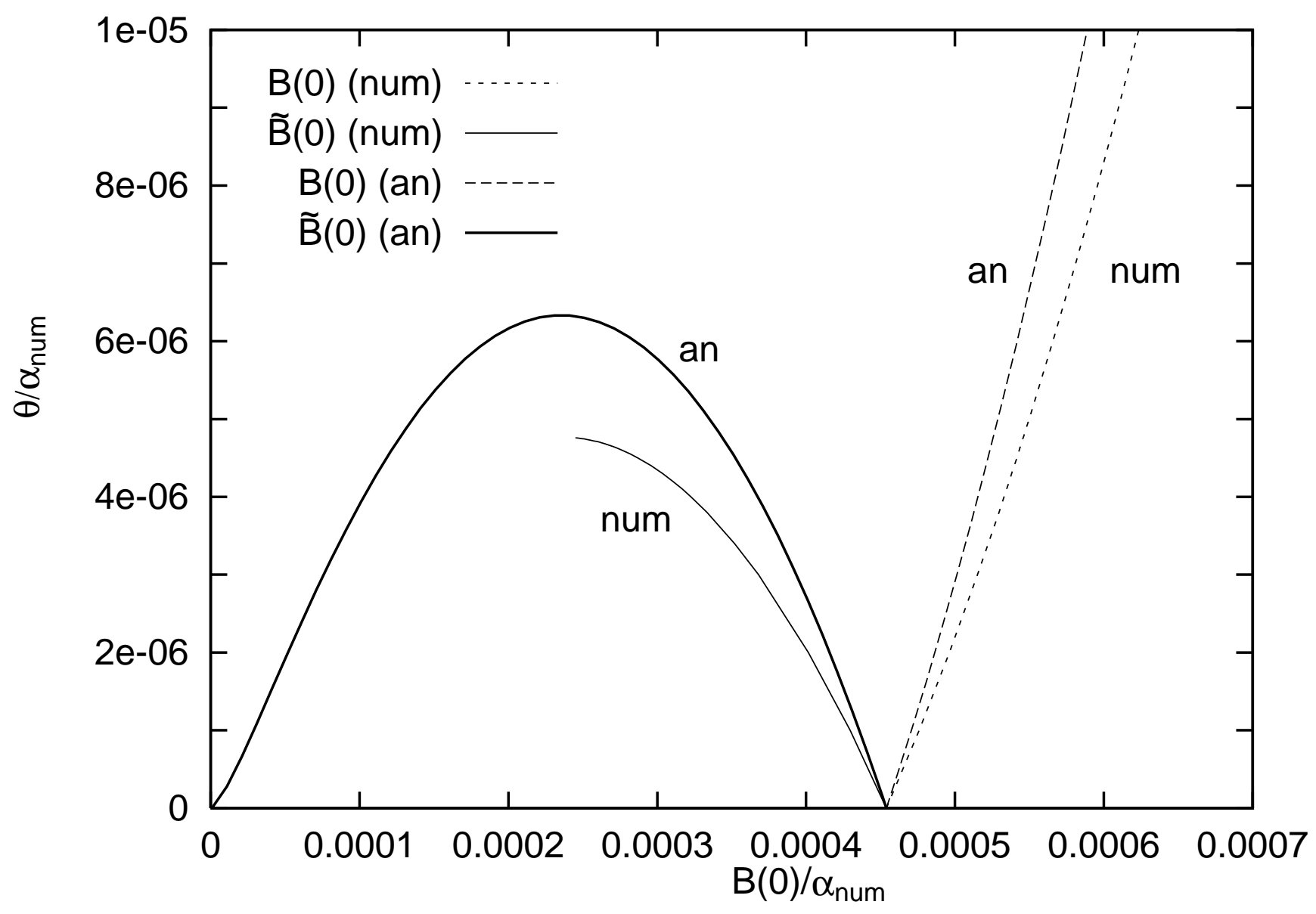


Figure $6 b$

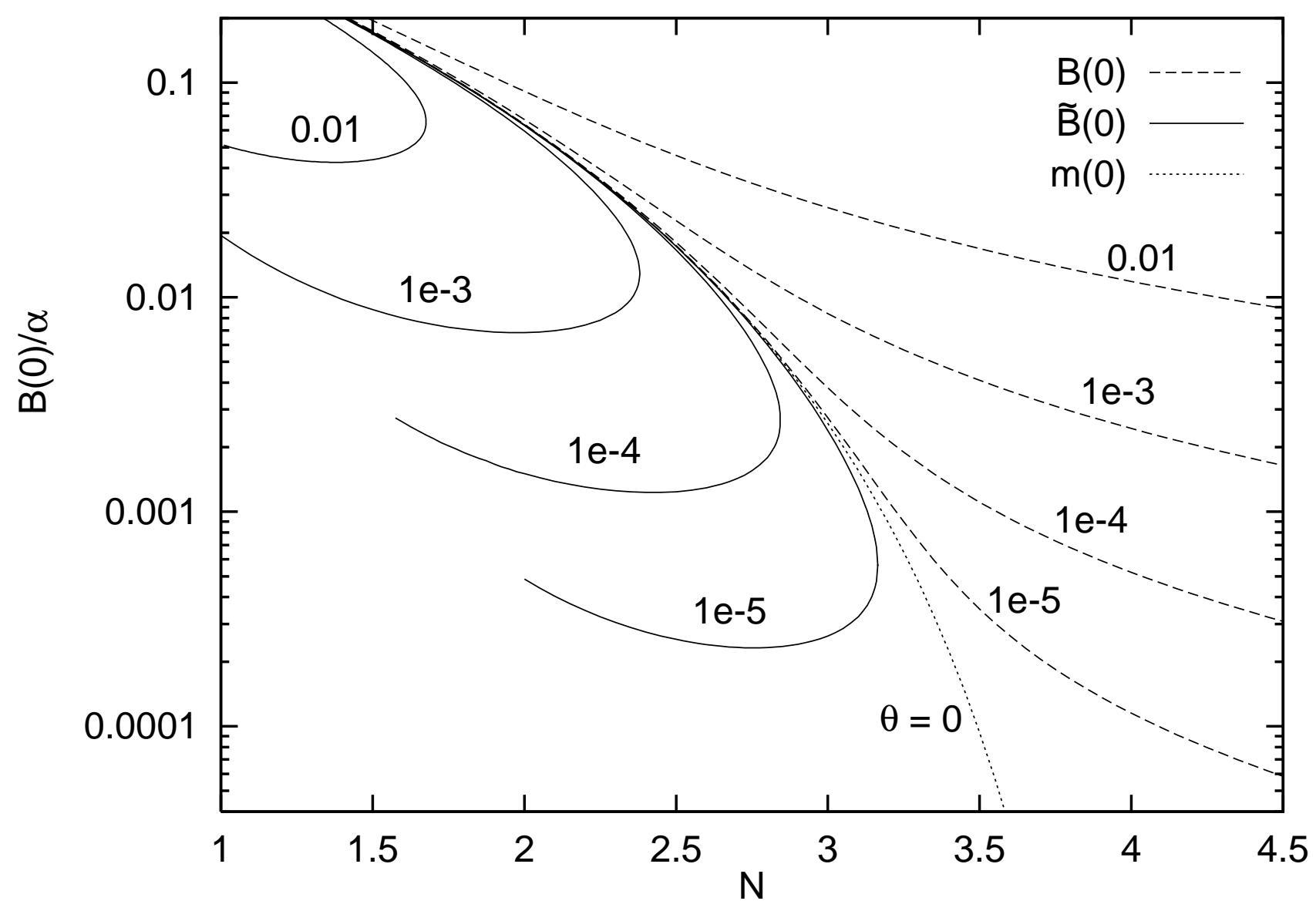


Figure 7

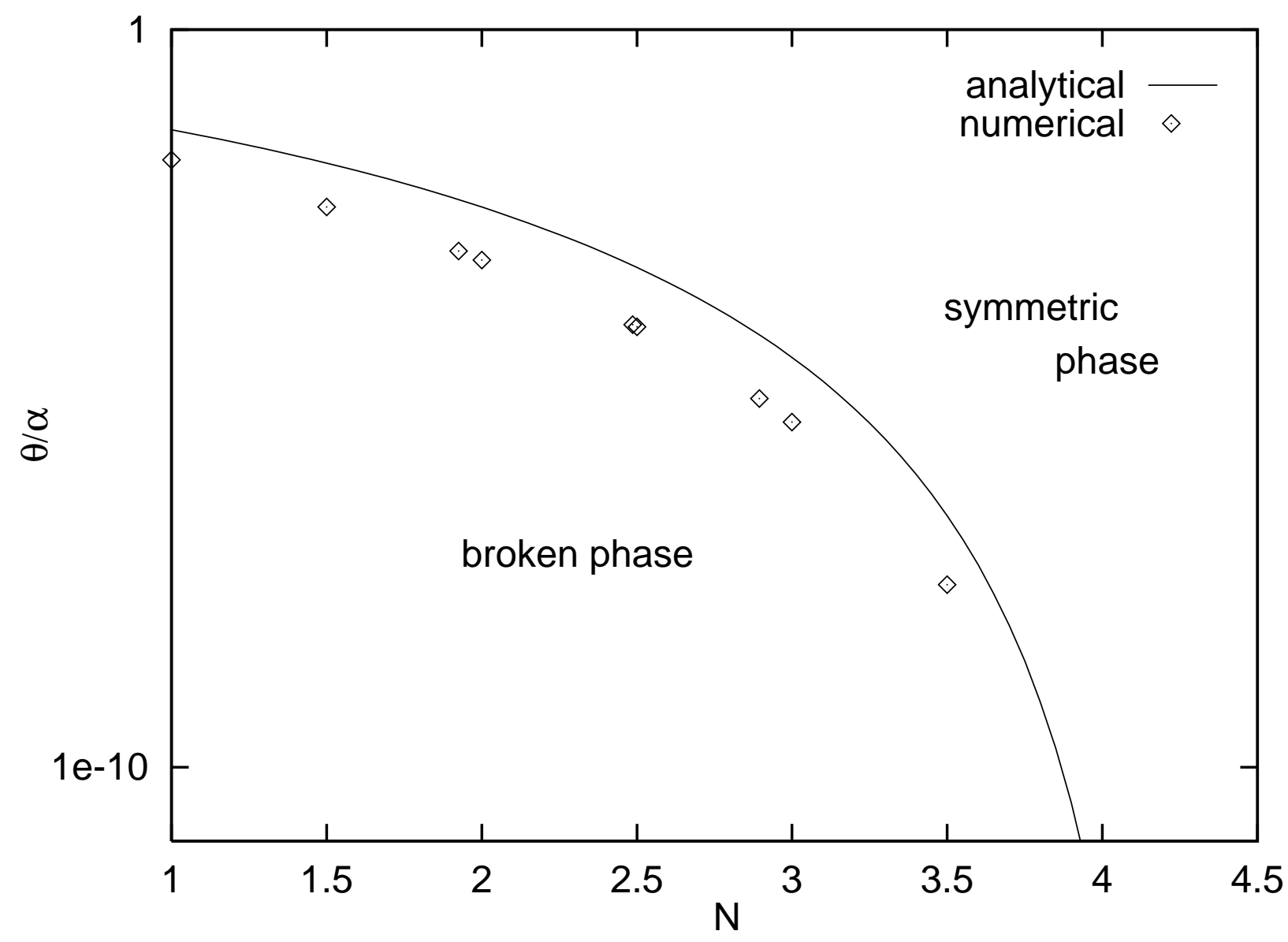


Figure 8

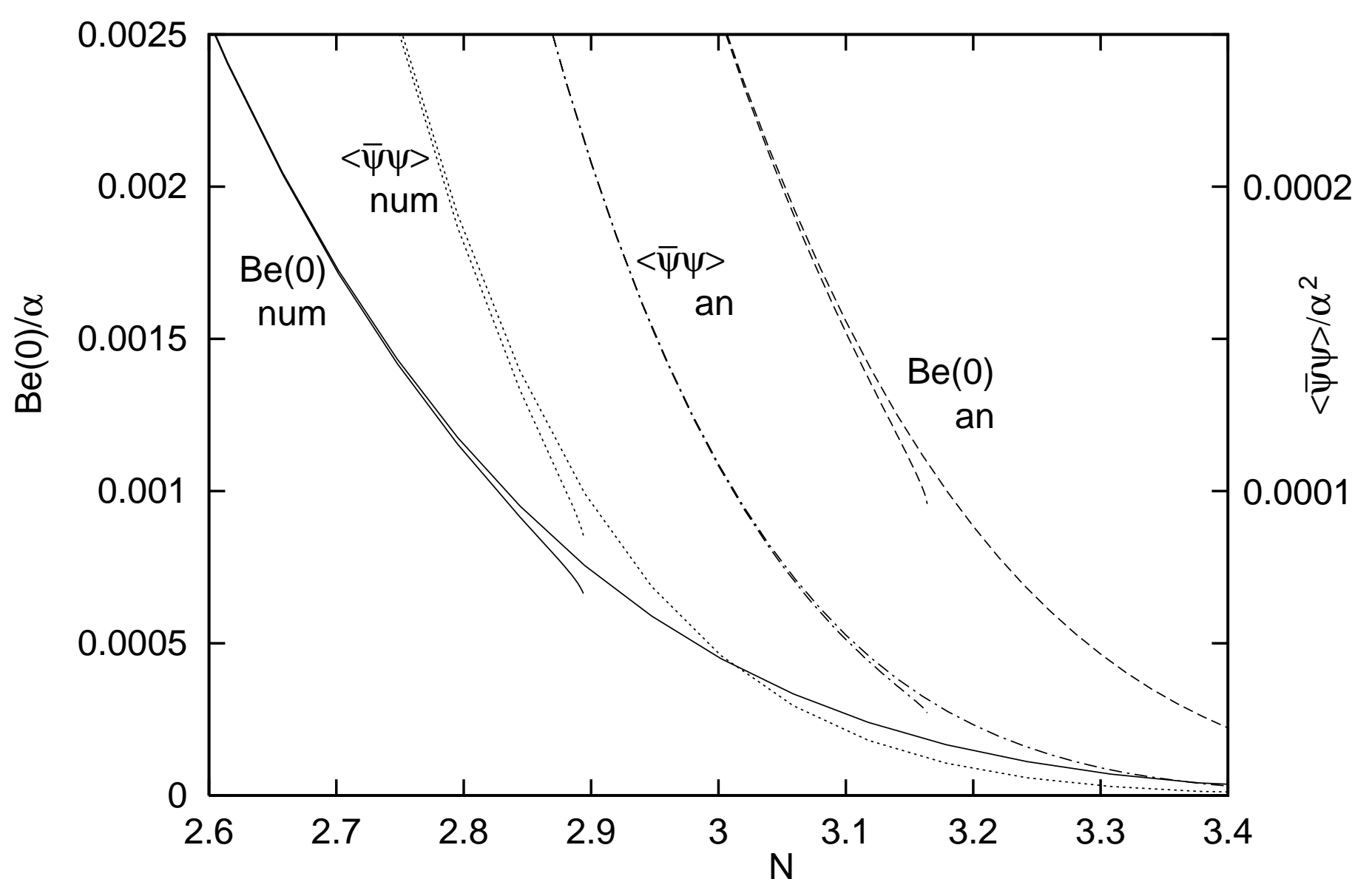


Figure 9

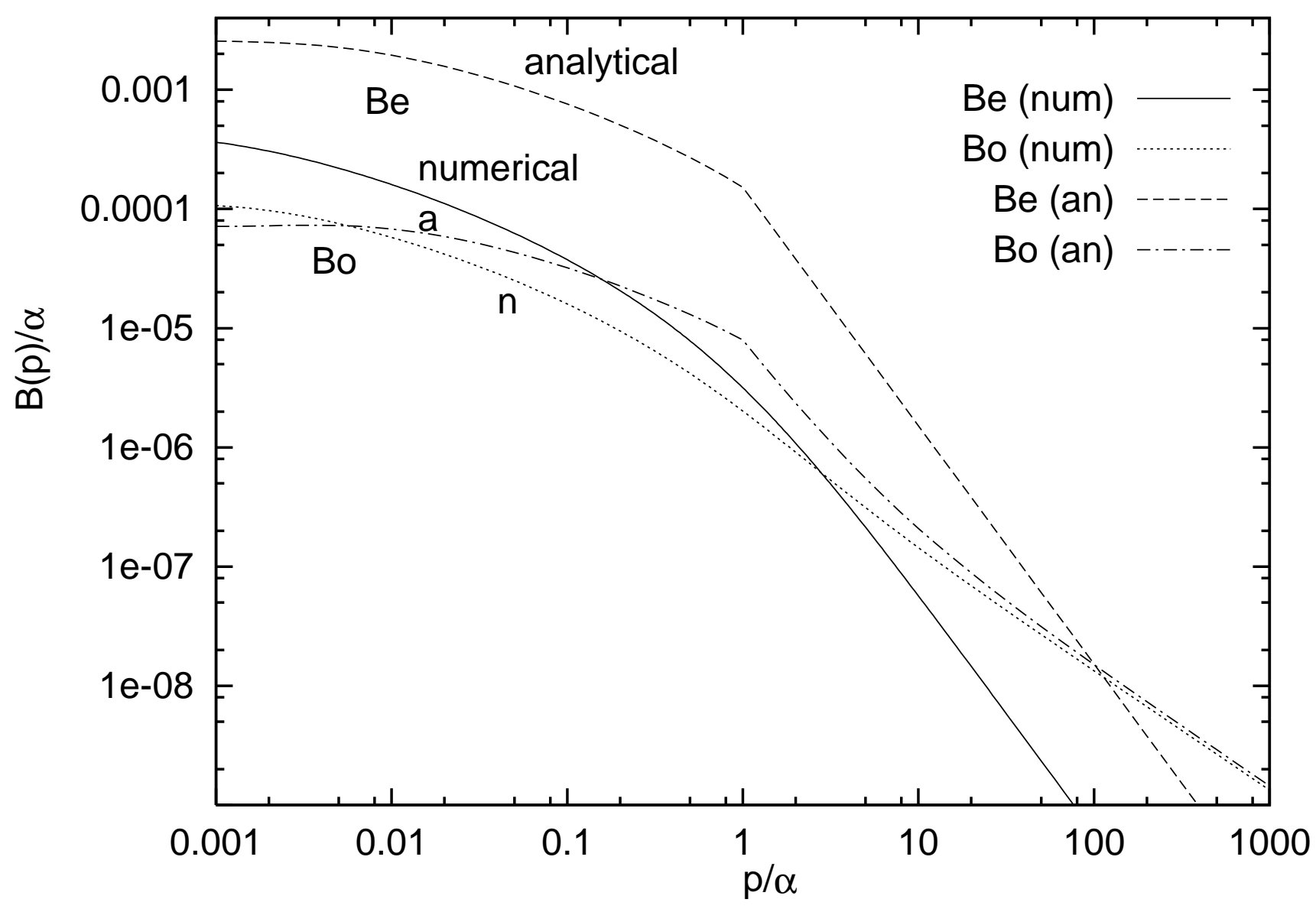

